\title{
Central Clearing Valuation Adjustment
}

\author{
Yannick Armenti and Stéphane Crépey* \\ stephane.crepey@univ-evry.fr \\ Université d'Évry Val d'Essonne \\ Laboratoire de Mathématiques et Modélisation d'Évry \\ 91037 Évry Cedex, France
}

February 6, 2017

\begin{abstract}
We develop an XVA (costs) analysis of the clearance framework for a member of a clearing house. The systemic consequences of the default of the clearing house itself are outside the scope of such an XVA analysis. Hence the clearing house is assumed default-free. We introduce a dynamic framework that incorporates the sequence of cash flows involved in the waterfall of resources of a clearing house. The overall XVA cost for a member, dubbed CCVA for central clearing valuation adjustment, is decomposed into CVA, MVA and KVA components. The CVA is the cost for a member of its losses on the default fund due to the defaults of other members. The MVA is the cost of funding initial margin. The KVA mainly consists in the cost of the capital at risk that the member provides to the CCP through its default fund contribution. In the end the structure of the XVA equations for bilateral and cleared portfolios is similar, but the data of the equations are of course not the same, reflecting the different financial network structures. The numerical experiments emphasize the multilateral netting benefit of central clearing. However, it is known that this multilateral netting comes at the expense of a loss of netting across asset classes. If we compensate the first order multilateral netting effect by a suitable scaling factor accounting for the loss of netting across asset classes, then the bilateral and centrally cleared XVA numbers become comparable. The second more explanatory factor of the numerical results is the credit risk of the members and the ensuing MVA, especially in the bilateral setup, where even more initial margin is required.
\end{abstract}

Keywords: Counterparty risk, central counterparty (CCP), margins, default fund, cost of funding, cost of capital, netting.

Mathematics Subject Classification: 60G44, 91B25, 91B26, 91B30, 91B70, 91B74, 91G20, 91G40, 91G60, 91G80,

JEL Classification: C63,C99 D52, D53,G12, G14, G21, G24, G28, G33.

* The research of Stéphane Crépey benefited from the support of the EIF grant "Collateral management in centrally cleared trading", of the "Chair Markets in Transition", Fédération Bancaire Française, and of the ANR 11-LABX-0019. 


\section{Introduction}

To cope with counterparty risk, the current trend in regulation is to push dealers to clear their trades via CCPs, i.e. central counterparties (also known as clearing houses). Progressively, central clearing is even becoming mandatory for vanilla products. Centrally cleared trading mitigates counterparty risk through an extensive netting of all transactions. Moreover, on top of the variation and initial margins that are used in the context of bilateral transactions, a CCP imposes its members to mutualize losses through an additional layer of protection, called the default or guarantee fund, which is pooled between the clearing members.

In this paper we develop the vision of a clearing house effectively eliminating counterparty risk (we do not incorporate the default of the clearing house in our setup), but at a certain cost for the members that we analyze. For this purpose, we develop an XVA (costs) analysis of centrally cleared trading, parallel to the one that has been developed in the last years for bilateral transactions.

\subsection{Review of the CCP Literature}

Duffie (2010) and Cont, Santos, and Moussa (2013) dwell upon the danger of creating "too big to fail" financial institutions, including, potentially, clearing houses.

Collateralization, whether in the context of centrally cleared trading or of bilateral trading under "standard CSA" (credit support annex), which is the emerging bilateral trading alternative to centrally cleared trading, requires a huge amount of cash or liquid assets. This puts a high pressure on liquidity, an issue addressed in Singh and Aitken (2009), Singh (2010), Levels and Capel (2012) and Duffie, Scheicher, and Vuillemey (2015). Relying on metrics à la Eisenberg and Noe (2001), Amini, Filipović, and Minca (2015) assess the systemic risk and incentivization properties of a CCP design where, in order to spare the clearing members from liquidation costs, in situations of financial distress, the clearing members could temporarily withdraw from their default fund contributions to post variation margin.

Avellaneda and Cont (2013) consider the optimal liquidation of the portfolio of a defaulted member by the clearing house.

Clearing is typically organized by asset classes, so that service closure of the CCP on one asset class does not harm its activity on other markets - and also because otherwise, in case of the default of a member, holders of less liquid assets (e.g. CDS contracts) are penalized with respect to holders of more liquid assets (e.g. interest rate swaps). As a consequence, the multilateral netting benefit of CCPs comes at the expense of a loss of bilateral netting across asset classes (see Duffie and Zhu (2011)). Cont and Kokholm (2014) claim that the former effect typically dominates the latter. But Ghamami and Glasserman (2016) show that, accounting for bilateral cross-asset netting, the higher regulatory capital and margin requirements adopted for bilateral contracts do not necessarily create the intended cost incentive in favor of central clearing.

Cont, Mondescu, and Yu (2011) and Pallavicini and Brigo (2013) analyze the pricing implications of the differences between the margining procedures involved in bilateral and centrally cleared transactions.

Until recently, the cost analysis of CCPs, our focus in this paper, was only considered in an old business finance literature reviewed in Knott and Mills (2002), notably Fenn and Kupiec (1993). In the last years, new papers have appeared in this direction. Under styl- 
ized assumptions, Arnsdorf (2012) derives an explicit approximation to a CCVA (using the terminology of the present paper), including effects such as wrong way risk (meant as procyclicality of the margins), credit dependence between members and left tailed distributions of their P\&Ls. Ghamami (2015) proposes a static one-period model where a CCVA can be priced by Monte-Carlo. Brigo and Pallavicini (2014) extend the bilateral counterparty risk dynamic setup of their previous papers to centrally cleared trading. However, they ignore the default fund and the credit risk dependence issues that are inherent to the position of a clearing member.

\subsection{Contributions and Outline}

This paper develops an XVA (costs) analysis of centrally cleared trading, parallel to the one that has been developed in the last years for bilateral transactions (see e.g. Crépey, Bielecki, and Brigo (2014, Parts II and III) or Brigo, Morini, and Pallavicini (2013)). A dynamic framework incorporates the sequence of cash flows involved in the waterfall of resources of the clearing house. As compared with Arnsdorf (2012) and Ghamami (2015), our CCVA accounts not only for the central clearing analog of the CVA, which is the cost for a member of its losses on the default fund in case of other members' defaults, but also for the cost of funding its margins (MVA) and for the cost of the capital (KVA) that is implicitly required from members through their default fund contributions (and for completeness and reference we also compute a DVA term).

The framework of this paper can be used by a clearing house to find the right balance between initial margins and default fund in order to minimize the CCVA (subject to the regulatory constraints), hence optimize its costs to the members for a given level of resilience. A clearing house can also use it to analyze the benefit for a dealer to trade centrally as a member rather than on a bilateral basis, or to help its members manage their CCVA (regarding the question for instance of how much of these costs they could consider passing to their clients).

The paper is organized as follows. Sect. 2 presents our clearing house setup. The waterfall of resources of the CCP is described in Sect. 3. The CCVA analysis is conducted in Sect. 4. Sect. 5 introduces the common shock model that is used for the default times of the members of the clearing house. Sect. 6 provides an executive summary of the centrally cleared XVA analysis of this paper and recalls for comparison purposes the bilateral CSA XVA methodology of Crépey and Song (2016), Sect. 7 designs an experimental framework used in the numerics of Sect. 8. Sect. 9 concludes. Regulatory formulas are recalled in Sect. A. Proofs of all lemmas are deferred to Sect. B.

\subsection{Basic Notation and Terminology}

$\int_{a}^{b}=\int_{(a, b]} ; x^{ \pm}=\max ( \pm x, 0) ; \boldsymbol{\delta}_{a}$ represents a Dirac measure at a point $a ; \boldsymbol{\lambda}$ denotes the Lebesgue measure on $\mathbb{R}_{+}$. Unless otherwise stated, a filtration satisfies the usual conditions; a price process is a special semimartingale in a càdlàg version; all inequalities between random quantities are meant almost surely or almost everywhere, as suitable; all the cash flows are assumed to be integrable whenever required; by "martingale" we mean local martingale unless otherwise stated, but true martingale is assumed whenever necessary. This means that we only derive local martingale properties. Usually in applications one needs true martingales, but this is not a real issue in our case, where even square integrability 
follows from additional assumptions postulated when dealing with BSDEs, which are our main pricing tool in this paper.

\section{Clearinghouse Setup}

We model a service of a clearing house dedicated to trading between its members, labeled by $i \in N=\{0, \ldots, n\}$.

\subsection{From Bilateral to Centrally Cleared Trading}

In a centrally cleared setup, the clearing house interposes itself in all transactions, becoming "the buyer to every seller and the seller to every buyer". All the transactions between the clearing house and a given member are netted together. See Figure 1 for an example, where the circled numbers in the left (respectively right) diagram show the gross positions of $n=3$ counterparties in a CSA setup (respectively their net positions with the CCP after the introduction of the latter in the middle).

In addition to interfacing all trades, the clearing house asks for several layers of guarantee to be posted by the members against counterparty risk, including a default fund that is pooled between the clearing members.

The benefits of centrally cleared trading are multilateral netting benefit and mutualization of risk. The drawbacks are an increase of systemic risk, where "too big to fail" CCPs might be created, liquidity risk, due to the margin requirements, and a loss of bilateral netting across asset classes (cf. Duffie (2010) and Cont et al. (2013)).
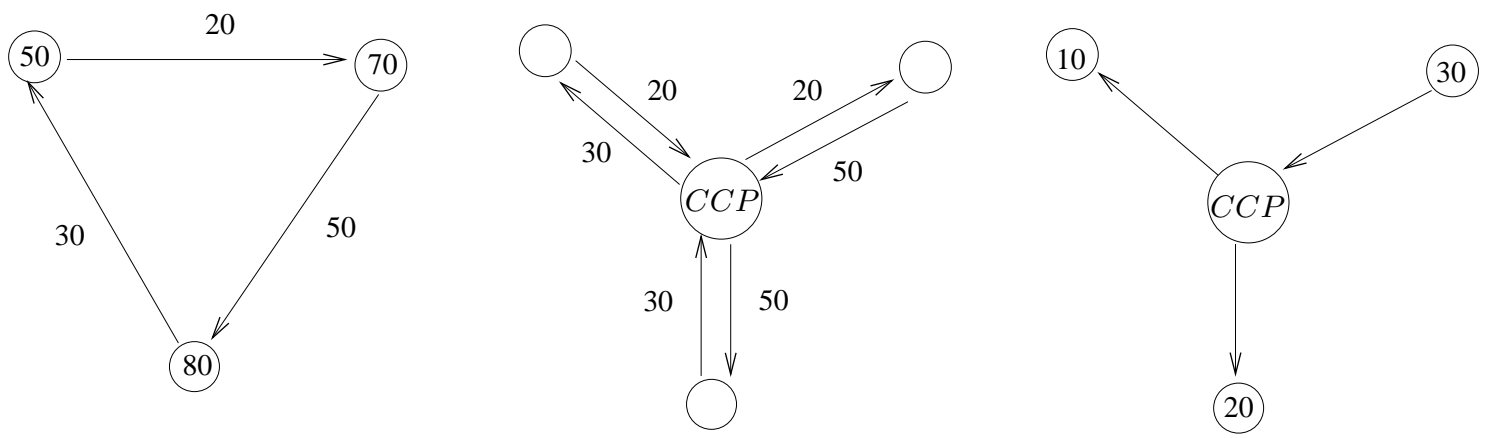

Figure 1: From bilateral to centrally cleared trading.

\subsection{Liquidation Procedure}

The mandate of a CCP is to liquidate over a few days the portfolio of a defaulted member. During the liquidation period, the CCP bears the risk of the portfolio. The trades with a defaulted member are typically reallocated by means of auctions among the surviving members and/or by a gradual liquidation of its assets in the market.

For ease of analysis in this paper, we assume the existence of a risk-free "buffer" that is used by the clearing house for replacing defaulted members in their transactions with others at the end of a liquidation period of length $\delta$ (the defaulted transactions already involving the buffer as one counterparty are simply terminated). We assume that during 
the liquidation period, the promised contractual cash flows and the hedge of a defaulted member are taken over by the CCP.

\subsection{Pricing Framework}

Let $(\Omega, \mathbb{G}, \mathbb{Q})$ represent a stochastic pricing basis, with $\mathbb{G}=\left(\mathcal{G}_{t}\right)_{t \in \mathbb{R}_{+}}$, such that all our processes are $\mathbb{G}$ adapted and all the random times of interest are $\mathbb{G}$ stopping times. Expectation under $\mathbb{Q}$ and $\left(\mathcal{G}_{t}, \mathbb{Q}\right)$ conditional expectation are denoted by $\mathbb{E}$ and $\mathbb{E}_{t}$. We denote by $r$ a $\mathbb{G}$ progressive OIS rate process and by $\beta_{t}=e^{-\int_{0}^{t} r_{s} d s}$ the corresponding discount factor. An OIS (overnight index swap) rate is together the best market proxy for a risk-free rate and the reference rate for the remuneration of the collateral.

For each member $i$, we denote by $D_{t}^{i}$ the process of the cumulative contractual cash flows of its portfolio with the CCP ("promised dividend" process ignoring counterparty and funding risk), assumed of finite variation. We denote by $P_{t}^{i}$ the mark-to-market of its portfolio ignoring counterparty and funding risk, i.e.

$$
\beta_{t} P_{t}^{i}=\mathbb{E}_{t}\left(\int_{t}^{\bar{T}} \beta_{s} d D_{s}^{i}\right), t \in[0, \bar{T}],
$$

where $\bar{T}$ is the final maturity of the CCP service portfolio, assumed held on a run-off basis (as is standard in any pricing or risk model). All cash flows and values are considered from the point of view of the clearing house, e.g. $P_{t}^{i}=1$ means that the member $i$ is short of a mark-to-market value equal to one (disregarding margins) toward the clearing house at time $t$. Since all trades are between the members, we have $\sum_{i \in N} P^{i}=0$.

\section{Margin Waterfall Analysis}

The mark-to-market pricing formula (2.1) ignores the counterparty risk of the member $i$, with default time $\tau_{i}$ and survival indicator process $J^{i}=\mathbb{1}_{\left[0, \tau_{i}\right)}$. As a first counterparty risk mitigation tool, the members are required to exchange variation margins that track the mark-to-market of their portfolios. A clearing house can call for variation margins at every time of a margin grid of step $h$, e.g. twice a day.

However, various frictions and delays, notably the liquidation period $\delta$, imply gap risk, which is the risk of a gap between the variation margin and the debt of a defaulted member at the time of liquidation of its portfolio. This is a special concern for certain classes of assets, such as credit derivatives, that may have quite unpredictable cash flows (see Crépey and Song (2016)).

This is why another layer of collateralization, called initial margins, is maintained in centrally cleared transactions as well as in bilateral transactions under standard CSA (the emerging bilateral trading alternative to centrally cleared trading). Initial margins are also dynamically updated, based on some risk measure of the variation-margined P\&L of each member computed over the time horizon $\delta^{\prime}=\delta+h$ of the so called margin period of risk (maximal time $h$ elapsed since the last margin call before the default plus liquidation period $\delta$ between default and liquidation).

Gap risk is magnified by wrong-way risk, which is the risk of adverse dependence between the positions and the credit risks of the members. One may also face credit contagion effects between members (wrong-way and contagion risk are of special concern regarding credit derivatives). Clearing houses deal with such extreme and systemic risk through a default 
fund mutualized between the clearing members. The default fund contribution of each member is primarily intended to reimburse the losses triggered by its own default, but, if rendered necessary by exhaustion of the previous layers of the waterfall, it can also be used for reimbursing the losses due to the defaults of other members.

\subsection{Margins}

Let $l h$, with $l \geq 0$, represent the times of the variation and initial margin calls, and let $l T$, with $T$ a multiple of $h$ (e.g. $h=$ one day and $T=$ one month), represent the times of update of the default fund contributions.

Consistent with our sign convention that all cash flows and values are seen from the perspective of the clearing house, we count a margin positively when it is posted by a member and we define the variation margin $V M^{i}$, initial margin $I M^{i}$ and default fund contribution $D F C^{i}$ of the member $i$ as the piecewise constant process reset at the respective grid times following, respectively (while the member $i$ is alive):

$$
V M_{l h}^{i}=P_{l h-}^{i}, \quad I M_{l h}^{i}=\rho_{l h}^{i}, \quad D F C_{l T}^{i}=\varrho_{l T}^{i},
$$

where $\rho^{i}$ and $\varrho^{i}$ refer to suitable risk measures as explained below. Note that (3.1) defines the level of reset of the respective cumulative amounts. Starting from $V M_{0}^{i}=P_{0-}^{i}, I M_{0}^{i}=\rho_{0}^{i}$ and $D F C_{0}^{i}=\varrho_{0}^{i}$, the corresponding updates at grid times are $\left(P_{l h-}^{i}-P_{(l h-h)_{-}}^{i}\right),\left(\rho_{l h}^{i}-\rho_{l h-h}^{i}\right)$ and $\left(\varrho_{l T}^{i}-\varrho_{l T-T}^{i}\right)$.

Remark 3.1 In practice, the variation margin only tracks the mark-to-market of the portfolio up to some thresholds, or free credit lines of the members, and up to minimal transfer amounts devoted to avoiding useless updates. These features, which can be important in the case of bilateral transactions, are omitted here as negligible in the case of centrally cleared transactions.

Let

$$
L_{t, t+\delta^{\prime}}^{i}=P_{t+\delta^{\prime}}^{i}+\int_{\left[t, t+\delta^{\prime}\right]} e^{\int_{s}^{t+\delta^{\prime}}} r_{u} d u d D_{s}^{i}-P_{t-}^{i} .
$$

In particular, at margin call times $t=l h$, we have, in view of the specification of the variation margin by the first identity in (3.1):

$$
L_{l h, l h+\delta^{\prime}}^{i}=P_{l h+\delta^{\prime}}^{i}+\int_{\left[l h, l h+\delta^{\prime}\right]} e^{\int_{s}^{l h+\delta^{\prime}} r_{u} d u} d D_{s}^{i}-V M_{l h}^{i}
$$

which is the variation-margined loss-and-profit of the member $i$ at the time horizon $\delta^{\prime}=$ $\delta+h$ of the margin period of risk (cumulative loss-and-profit also accounting for all the contractual cash flows capitalized at the risk-free rate during the margin period of risk $\left.\left[t, t+\delta^{\prime}\right]\right)$. The risk measure used for fixing the initial margins is a univariate risk measure computed at the level of each member individually, which we write as

$$
\rho_{l h}^{i}=\rho\left(L_{l h, l h+\delta^{\prime}}^{i}\right)
$$

where $\rho$ can be value at risk, expected shortfall, etc.. The dependence between the portfolios of the members is only reflected in the initial margins through the structural constraint that $\sum_{i \in N} P^{i}=0$. 
Remark 3.2 Historically, for computing initial margins, CCPs have been mostly using the SPAN methodology, introduced by the Chicago Mercantile Exchange in the 80s. This methodology is based, for each member, on the consideration of the most unfavorable among sixteen reference scenarios (see Kupiec and White (1996)). Nowadays, value at risk methodologies tend to become the standard.

Unless defaults happen, margins do not imply any transfer of ownership and can be seen in this sense as a loan by the posting member. By contrast, default fund contributions can be consumed in case of other members' defaults, hence they should really be viewed as capital put at the disposal of the CCP by the clearing members. The "cover two" EMIR rule prescribes to size the default fund as, at least, the maximum of its largest exposure and of the sum of its second and third largest exposures to the clearing members (see Sect. A.1). This is only a regulatory minimum and sometimes more conservative rules are used, such as a default fund set as the sum of the two largest exposures. It is then allocated between the clearing members by some rule, e.g. proportionally to their initial margins. At a more theoretical level, the mutualization rationale of the default fund calls for the use of multivariate risk measures, which we write in an abstract fashion as

$$
\varrho_{l T}^{i}=\varrho_{i}\left(\left(L_{l T, l T+\delta^{\prime}}^{j}-I M_{l T}^{j}\right)_{j ; J_{l T}^{j}=1}\right)
$$

(or an analog formula involving not only the $L_{l T, l T+\delta^{\prime}}^{j}$, but also intermediary $L_{\cdot, \cdot+\delta^{\prime}}^{j}$ between $(l-1) T$ and $l T$ to refrain members from temporarily closing their positions right before $l T$ in order to avoid to contribute to the default fund).

Regarding the distributions that are used for members loss-and-profits in all these risk measure computations, since the crisis, the focus has shifted from the cores of the distributions, dominated by volatility effects, to their queues, dominated by scenarios of crisis and default events. For the determination of the initial margins, Gaussian VaR models are generally banned since the crisis and CCPs typically focus on either Pareto laws or on historical VaR. Stressed scenarios and parameters are used for the determination of the default fund.

Note that margin schemes as above, even, in the case of the default fund contributions, possibly based on multivariate risk measures (cf. (3.5)), only account for asset dependence between the portfolios of the members, ignoring credit risk and contagion effects between members. This is in line with the mandate of a clearing house to mitigate (i.e. put a cap on) its exposure to the members by means of the margins, in case a default would happen, where a defaults is viewed as a totally unpredictable event. On top of the margins, add-ons are sometimes required from members with particularly high credit or concentration risk.

We refer the reader to Ghamami (2015), Cruz-Lopez, Harris, Hurlin, and Perignon (2015), Menkveld (2016) or Armenti, Crépey, Drapeau, and Papapantoleon (2015) for alternative margin schemes and default fund specifications. Good margining schemes should guarantee the required level of resilience to the clearing house at a bearable cost for the members. Two points of concern are procyclicality, in particular with haircuts that increase with the distress of a member, and liquidity, given the generalization of central clearing and collateralization. Capponi and Cheng (2016) construct a model which endogenizes collateral, making it part of an optimization problem where the CCP maximizes profit by controlling collateral and fee levels. 


\subsection{Breaches}

The default time of the member $i$ is modeled as a stopping time $\tau_{i}$ with an intensity process $\gamma^{i}$. In particular, any event $\left\{\tau_{i}=t\right\}$, for a fixed time $t$, has zero probability and can be ignored in the analysis. For every time $t \geq 0$, let

$$
\bar{t}=t \wedge \bar{T}, t^{\delta}=t+\delta, \quad \bar{t}^{\delta}=\mathbb{1}_{t<\bar{T}} t^{\delta}+\mathbb{1}_{t \geq \bar{T}} \bar{T}
$$

and let $\widehat{t}$ denote the greatest margin call time $l h \leq t$. We denote by

$$
\mathcal{C}^{i}=V M^{i}+I M^{i}+D F C^{i}
$$

the overall collateral process of the member $i$. We assume that collateral posted is remunerated OIS and that the CCP substitutes itself to a defaulted member during its liquidation period, including regarding these collateral OIS remuneration cash flows. In our model collateral earns OIS but collateral OIS earnings are transferred as a remuneration to the posting member, they do not stay in the collateral accounts. Hence, the amount of available collateral for the liquidation of a defaulted member does not accrue at the OIS rate but stays constant during the liquidation period. As a consequence, we have $\mathcal{C}_{t}^{i}=\mathcal{C}_{\widehat{t}}^{i}$ for $t \leq \tau_{i}$ and the process $\mathcal{C}$ is stopped at time $\widehat{\tau}_{i}$. For each member $i$, we write

$$
\begin{aligned}
& \Delta_{t}^{i}=\int_{\left[\tau_{i}, t\right]} e^{\int_{s}^{t} r_{u} d u} d D_{s}^{i}, \quad Q_{t}^{i}=P_{t}^{i}+\Delta_{t}^{i}, \quad \varepsilon_{i}=\left(Q_{\tau_{i}^{\delta}}^{i}-\mathcal{C}_{\widehat{\tau}_{i}}^{i}\right)^{+}, \\
& \chi_{i}=-\mathbb{1}_{\varepsilon_{i}=0} Q_{\tau_{i}^{\delta}}^{i}-\mathbb{1}_{\varepsilon_{i}>0}\left(\mathcal{C}_{\widehat{\tau}_{i}}^{i}+R_{i} \varepsilon_{i}\right), \\
& \xi_{i}=Q_{\tau_{i}^{\delta}}^{i}+\chi_{i}=\mathbb{1}_{\varepsilon_{i}>0}\left(Q_{\tau_{i}^{\delta}}^{i}-\mathcal{C}_{\widehat{\tau}_{i}}^{i}-R_{i} \varepsilon_{i}\right)=\left(1-R_{i}\right) \varepsilon_{i},
\end{aligned}
$$

where $\Delta_{t}^{i}$ represents the cumulative contractual dividends capitalized at the risk-free rate that fail to be paid by member $i$ from time $\tau_{i}$ onwards. These dividends are promised but unpaid due to the default of the member $i$ at $\tau_{i}$. Hence, they also belong to the exposure of the CCP to the default of the member $i$. More precisely, as will be understand in more detail from the proof of Lemma 3.1 below, $\chi_{i}$ corresponds to a terminal cash flow closing the position of the defaulted member $i$, paid by the CCP to the estate of the defaulted member at time $\tau_{i}^{\delta} ; \varepsilon_{i}$ corresponds to the raw exposure of the CCP to the default of the member $i$; $\xi_{i}$ is the exposure accounting for an assumed recovery rate $R_{i}$ of the member $i$. In fact, in the context of centrally cleared trading, by liquidation of a defaulted member, we simply mean the liquidation of its CCP portfolio, as opposed to the legal liquidation, by a mandatory liquidator, that can take several years (the New York branch of Lehman was legally liquidated in December 2013, more than five years after Lehman's default). In particular, there is typically no recovery to expect on a defaulted member, i.e. $R_{i}=0$. The reason why we introduce recovery coefficients is for the discussion regarding DVA and DVA2 in Sect. 4 and for comparison with the bilateral trading setup of Sect. 6.

Note that we do not exclude joint defaults in our setup. In fact, joint defaults, which can be viewed as a form of "instantaneous contagion", is the way we will introduce credit dependence between members in Sect. 5. For $Z \subseteq N=\{0, \ldots, n\}$, we denote by $\tau_{Z} \in$ $\mathbb{R}_{+} \cup\{\infty\}$ the time of joint default of names in the subset $Z$ and only in $Z$. At this stage we consider all the costs from the perspective of the CCP and the community of the surviving members altogether. The allocation of these costs between the CCP and the surviving members will be considered in Sect. 3.3. We call realized breach of a (possibly joint) default event the residual loss to the CCP after all the collateral of the defaulted member(s) has been consumed. 
Lemma 3.1 At each liquidation time $\tau_{Z}^{\delta}=\tau_{Z}+\delta$ with $\tau_{Z}<\bar{T}$, the realized breach of the $C C P$ is given by

$$
B_{\tau_{Z}^{\delta}}=\sum_{i \in Z} \xi_{i}
$$

\subsection{Equity and Default Fund Replenishment Principle}

We proceed with the description of the next layers of the waterfall of resources of the clearing house, namely the equity and the default fund.

If the default of a member entails a positive breach, then the first payer (although to a typically quite limited extent) is the clearing house itself (before the surviving members), via its equity $E$.

Remark 3.3 The regulation (e.g. EMIR) does not necessarily require that the CCP be the first payer in case of a realized breach. However, CCPs typically take the equity tranche of this risk, as a good management incentive.

Specifically, at times $l Y, l \geq 0$, where $Y$ is a multiple of $T$ (e.g. one year whereas $T$ is one month), the equity process $E$ is reset by the clearing house at some target level $E_{l Y}^{\star}$, the "skin in the game" of the clearing house for the time period $[l Y,(l+1) Y]$. In the meantime, the equity is used as first resource for covering the realized breaches, i.e., at each $t=\tau_{Z}^{\delta}$ with $\tau_{Z}<\bar{T}$, we have

$$
\Delta E_{t}=-\left(B_{t} \wedge E_{t-}\right)
$$

The part of the realized breach left uncovered by the equity, $\left(B_{t}-E_{t-}\right)^{+}$, is covered by the surviving members through the default fund, which they refill instantaneously by the following rule, at each $t=\tau_{Z}^{\delta}$ with $\tau_{Z}<\bar{T}$ (see Figure 2):

$$
\epsilon_{t}^{i}=\left(B_{t}-E_{t-}\right)^{+} \frac{J_{t}^{i} D F C_{t}^{i}}{\sum_{j \in N} J_{t}^{j} D F C_{t}^{j}},
$$

proportionally to their current default fund contributions $D F C_{t}^{i}$ (or other keys of repartition such as their initial margins or the notionals of their positions).

In sum, the margins and the default fund contributions $V M_{l h}^{i}, I M_{l h}^{i}$ and $D F C_{l T}^{i}$ are reset at their respective grid times by the surviving members according to (3.1); the equity is reset at the times $l Y$ by the clearing house and is used for covering the first levels of realized breaches at liquidation times according to (3.10); the losses in case of realized breaches above the residual equity are covered at liquidation times by the surviving members according to (3.11) (see Figure 2).

Remark 3.4 The total size of the default fund is $\sum_{j \in N} J^{j} D F C^{j}$, a quantity also referred to as the funded default fund. The unfunded default fund refers to the additional amounts members may have to pay via the above default fund replenishment principle in case of defaults of other members. More precisely,

$$
u_{l T}^{i}=\left(\sum_{l T-T<\tau_{Z}^{\delta}<l T} \epsilon_{\tau_{Z}^{\delta}}^{i}-D F C_{l T-T}^{i}\right)^{+}
$$




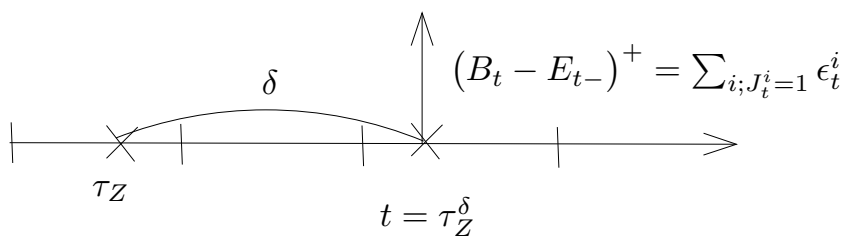

Figure 2: Margin cash flows: resets at margin call grid times and refill of the default fund at liquidation times.

represents the unfunded default fund contribution of the member $i$ for the period (lT $T, l T)$. The service closure, i.e. the closure of the activity of the clearing house on a given market or service, is usually specified in terms of events such as the unfunded default fund $\sum_{j \in N} J_{l T}^{j} u_{l T}^{j}$ reaching a cap given as, e.g., $2 \sum_{j \in N} J_{l T-T}^{j} D F C_{l T-T}^{j}$, i.e. twice the funded default fund. Given the high levels of initial margins that are used in practice, this is a very extreme tail event. Moreover, in case of service closure, the risk of a member is bounded above by the sum between its margins, three times its default fund contribution (assuming the above specification of service closure) and the cost of the liquidation of the service for this member. This cost is itself bounded by the notional of the member position, which would only be the actual cost in a scenario where all the assets of the CCP would jump to zero, also a very unlikely situation. In conclusion, the service closure event does not really matter regarding our present purpose of the XVA cost analysis of CCP membership. The default of the CCP as a whole (i.e. the closure of all its services) is an even more unlikely event, especially because a central bank would hardly allow it to occur in view of its systemic consequences. Hence we may and do ignore the service closure and the default of the clearing house in the context of this paper. See Armakola and Laurent (2015) about CCP resilience and see Duffie (2014) about alternative approaches to the design of insolvency and failure resolution regimes for CCPs.

\section{Central Clearing Valuation Adjustment}

We refer to the (generic) member 0 as "the member" henceforth, the other members being collectively referred to as "the clearing house". For notational simplicity, we remove the index 0 referring to the reference member. We call value of the $\mathrm{CCP}$ portfolio of the member its value inclusive of counterparty and funding risk (as opposed to the mark-to-market of the portfolio).

We assume that the member enters its portfolio at time 0 , against an upfront payment of a certain amount $\Pi_{0}$, where the semimartingale $\Pi$ is a tentative value process of the CCP portfolio of the member. We assume that profit-and-losses are marked to the model value process $\Pi$ and realized in continuous time (the reader is referred to Albanese et al. (2016, Section 9.1) for the discussion of other choices in this regard).

In this section, we derive a representation of the (no arbitrage) value $\Pi$ of the CCP portfolio of a member as the difference (cf. the remark 4.2 below) between the markto-market of the portfolio and a correction $\Theta$. We call $\Theta$ the central clearing valuation adjustment (CCVA). The KVA-inclusive CCVA is obtained in a second step by adding to $\Theta$ a capital valuation adjustment (KVA) meant as the cost that it would require for remunerating the member at some hurdle rate for its CCP capital at risk (including its 
default fund contribution).

\subsection{DVA and DVA2 Issues}

From the perspective of the member, the effective time horizon of interest is $\bar{\tau}^{\delta}$ (cf. (3.6)). The position of the member is closed at $\tau^{\delta}$ (if $\tau<\bar{T}$ ), with a terminal cash flow from the member's perspective given, in view of $(3.8)$ and of the analysis developed in the proof of Lemma 3.1 (for $i=0$ here), by

$$
\chi=-\mathbb{1}_{\varepsilon=0} Q_{\tau^{\delta}}-\mathbb{1}_{\varepsilon>0}\left(\mathcal{C}_{\widehat{\tau}}+R \varepsilon\right) .
$$

In particular, if $\varepsilon>0$, i.e. $Q_{\tau^{\delta}}>\mathcal{C}_{\widehat{\tau}}$, then the member receives

$$
-\mathcal{C}_{\widehat{\tau}}-R \varepsilon=-\mathcal{C}_{\widehat{\tau}}-R\left(Q_{\tau^{\delta}}-\mathcal{C}_{\widehat{\tau}}\right)=\left(-Q_{\tau^{\delta}}\right)+(1-R)\left(Q_{\tau^{\delta}}-\mathcal{C}_{\widehat{\tau}}\right)
$$

However, for this amount to benefit to the member's shareholders, it needs to be hedged so that they can monetize it before $\tau$ (otherwise it is only a profit to the member's bondholders). But, in order to hedge this amount, the member would basically need to sell credit protection on itself, which is barely possible in practice. Consequently, from an entry (i.e. transaction) price perspective, the member should ignore such a windfall benefit at own default and the ensuing debt valuation adjustment (DVA). This means formally setting $R=1$, which results in $\chi=-Q_{\tau^{\delta}}$ in (4.1) and $\xi=0$ later in 4.9). Then $R$ becomes disconnected from what the clearing house would actually recover (if anything) from the member in case it defaults, but this is immaterial for analyzing the costs of this member itself, it only matters for the others. In sum, it is possible and convenient to analyze the no DVA case for the reference member just by formally setting $R=1$.

If, however, some DVA is accounted for (i.e. if $R<1$ ), then one may want to reckon likewise a funding benefit of the member at its own default, a windfall benefit called DVA2 in the terminology of Hull and White (2012), corresponding to an additional cash flow to the member of the form

$$
(1-\bar{R})\left(\Pi_{\tau-}+\mathcal{C}_{\widehat{\tau}}^{\star}\right)^{+}
$$

at time $\tau$ (if $<\bar{T}$ ). Here $\mathcal{C}^{\star}=V M+I M$ and $\bar{R}$ is a recovery rate of the member to its funder, so that the amount $\left(\Pi_{\tau-}+\mathcal{C}_{\widehat{\tau}}^{\star}\right)^{+}$in 4.2 represents the funding debt of the member at its default (having assumed profit-and-losses marked-to-model and realized in real time, see the proof of Lemma 4.1 below for more detail). The funder of the member corresponds to a third party, possibly composed in practice of several entities or devices and assumed default-free for simplicity, playing the role of lender/borrower of last resort after exhaustion of the internal sources of funding provided to the member through its collateral and its hedge.

More generally, even if one considers that the "true" recovery rate of the member is simply zero, playing with formal recovery coefficients $R$ and $\bar{R}$ somewhere between 0 and 1 allows reaching any desired level of interpolation between the entry price point of view

$R=\bar{R}=1$ and the reference exit price point of view $R=\bar{R}=0$. On the DVA and DVA2 issues, see Hull and White (2012), Burgard and Kjaer (2012), Albanese and Andersen (2015), Albanese, Andersen, and Iabichino (2015), Andersen, Duffie, and Song (2016) and Albanese et al. (2016), 


\subsection{Gain Process}

The member can hedge its collateralized portfolio and needs to fund its whole position (portfolio, margins and hedge).

Regarding hedging, we restrict ourselves to the situation of a fully securely funded hedge, entirely implemented by means of swaps, short sales and repurchase agreements (all traded outside the clearing house, given our assumption of a constant CCP portfolio of the member), at no upfront payment. As explained in Crépey et al. (2014, Section 4.2.1 page 87 ), this assumption encompasses the vast majority of hedges that are used in practice. Consistent with arbitrage requirements and our terminology of a risk-neutral measure $\mathbb{Q}$, we assume that the vector-valued gain process $\mathcal{M}$ of unit positions in the hedging assets is a $\mathbb{Q}$ martingale (see Crépey et al. (2014, Remark 4.4.2 pages 96-97) or Bielecki and Rutkowski $(2015$, Proposition 3.3)). We assume that the member sets up a related hedge $(-\zeta)$, i.e. a predictable row-vector process with components yielding the (negative of) positions in the hedging assets. The "short" negative notation in front of $\zeta$ is used for consistency with the idea, just to fix the mindset, that the portfolio is "bought" by the member, which therefore "sells" the corresponding hedge.

Regarding funding, we assume that variation margins $V M_{t}=P_{\widehat{t}-}$ consist of cash rehypothecable and remunerated at OIS rates, while initial margins consist of segregated liquid assets accruing at OIS rates. Initial margins and default fund contributions are supposed to be subject to CCP fees $c_{t}$, e.g. 30 basis points. We postulate that the member can invest excess-cash at a rate $\left(r_{t}+\lambda_{t}\right)$ and obtain unsecured funding at a rate $\left(r_{t}+\bar{\lambda}_{t}\right)$.

Let $e$ denote the gain process (or profit-and-loss, hedging error,..) of the member's position, held by the member itself before $\bar{\tau}$ and then, if $\tau<\bar{T}$, by the clearing house (as liquidator of the member's position) on $\left[\bar{\tau}, \bar{\tau}^{\delta}\right]$.

Lemma 4.1 We have $e_{0}=0$ and, for $0<t \leq \bar{\tau}^{\delta}$,

$$
\begin{aligned}
& d e_{t}= d \Pi_{t}-r_{t} \Pi_{t} d t-J_{t}\left(d D_{t}+\sum_{Z \subseteq N} \epsilon_{\tau_{Z}^{\delta}} \boldsymbol{\delta}_{\tau_{Z}^{\delta}}(d t)+g_{t}\left(\Pi_{t}\right) d t\right) \\
&-\mathbb{1}_{\{\tau<\bar{T}\}}(1-\bar{R})\left(\Pi_{t-}+\mathcal{C}_{\widehat{t}}^{\star}\right)^{+} d J_{t}-\zeta_{t} d \mathcal{M}_{t},
\end{aligned}
$$

where, for any $\pi \in \mathbb{R}$,

$$
g_{t}(\pi)=c_{t}\left(\mathcal{C}_{t}-P_{\widehat{t}-}\right)+\bar{\lambda}_{t}\left(\pi+\mathcal{C}_{t}^{\star}\right)^{+}-\lambda_{t}\left(\pi+\mathcal{C}_{t}^{\star}\right)^{-} .
$$

Remark 4.1 The self-financing equation (4.3) holds for any funding coefficient $g_{t}=g_{t}(\pi)$ there, not necessarily given by (4.4), as soon as $\left(r_{t} \Pi_{t}+g_{t}\left(\Pi_{t}\right)\right) d t$ represents the $d t$-funding cost of the member (whilst the member is alive, and net of the funding cost of its hedge that is already comprised in the local martingale $\left.\zeta_{t} d \mathcal{M}_{t}\right)$.

\subsection{Pricing BSDE}

Definition 4.1 We call $\Pi$ a (no arbitrage) value process for the member's portfolio if $\Pi_{\bar{\tau}^{\delta}}=\mathbb{1}_{\{\tau<\bar{T}\}} \chi$ and the ensuing gain process $e$ (cf. 4.3) ) is a risk-neutral local martingale. 
Proposition 4.1 A semimartingale $\Pi$ is a value process for the member's portfolio if and only if it satisfies the following valuation BSDE on $\left[0, \bar{\tau}^{\delta}\right]$ :

$$
\begin{aligned}
\Pi_{\bar{\tau}^{\delta}}= & \mathbb{1}_{\{\tau<\bar{T}\}} \chi \text { and, for } t \leq \bar{\tau}^{\delta}, \\
d \Pi_{t}= & r_{t} \Pi_{t} d t+\mathbb{1}_{\{\tau<\bar{T}\}}(1-\bar{R})\left(\Pi_{t-}+\mathcal{C}_{\hat{t}}^{\star}\right)^{+} d J_{t} \\
& \quad+J_{t}\left(d D_{t}+\sum_{Z \subseteq N} \epsilon_{\tau_{Z}^{\delta}} \boldsymbol{\delta}_{\tau_{Z}^{\delta}}(d t)+g_{t}\left(\Pi_{t}\right) d t\right)+d \nu_{t},
\end{aligned}
$$

for some local martingale $\nu$.

Proof. In view of (4.3), (4.5) is equivalent to $d e_{t}=d \nu_{t}-\zeta_{t} d \mathcal{M}_{t}$. Since $\zeta_{t} d \mathcal{M}_{t}$ defines a local martingale, therefore $e$ and $\nu$ are jointly local martingales or not, which establishes the proposition.

Note that, assuming $\nu$ a true martingale, equivalently to the differential formulation (4.5), we can write (absorbing the $r_{t} \Pi_{t} d t$ term from (4.5) into the risk-neutral discount factor $\beta$ in (4.6) :

$$
\begin{aligned}
\beta_{t} \Pi_{t}= & \mathbb{E}_{t}\left[\mathbb{1}_{\{\tau<\bar{T}\}}\left(\beta_{\tau^{\delta}} \chi+\beta_{\tau}(1-\bar{R})\left(\Pi_{\tau-}+\mathcal{C}_{\widehat{\tau}}^{\star}\right)^{+} J_{t}\right)\right. \\
& \left.-\sum_{t<\tau_{Z}^{\delta}<\bar{\tau}} \beta_{\tau_{Z}^{\delta}} \epsilon_{\tau_{Z}^{\delta}}-\int_{t}^{\bar{\tau}} \beta_{s} J_{s}\left(d D_{s}+g_{s}\left(\Pi_{s}\right) d s\right)\right], 0 \leq t \leq \bar{\tau}^{\delta} .
\end{aligned}
$$

\subsection{CCVA Representation}

In this section we define the central counterparty valuation adjustment (CCVA) and derive the corresponding BSDE.

Definition 4.2 Given a value $\Pi$ for the member, the corresponding CCVA is the process defined on $\left[0, \bar{\tau}^{\delta}\right]$ as $\Theta=-(Q+\Pi)$.

Remark 4.2 Recall from (3.8) that $Q=P+\Delta$, with all values viewed from the perspective of the clearing house. Consistent with the usual definition of a valuation adjustment (see Brigo et al. (2013) or Crépey et al. (2014)), we have $\Theta=(-Q)-\Pi$, where $(-Q)$ corresponds to the perspective of the member.

Let

$$
\bar{\xi}_{t}=\mathbb{E}\left(\beta_{t}^{-1} \beta_{\tau+\delta} \xi \mid \mathcal{G}_{t}\right),
$$

where $\xi=(1-R)\left(Q_{\tau^{\delta}}-\mathcal{C}_{\widehat{\tau}}\right)^{+}$as before $(\mathrm{cf}$. $(3.8))$. Let $\widehat{\xi}$ be a $\mathbb{G}$ predictable process, which exists by Corollary 3.23 2) in He, Wang, and Yan (1992), such that

$$
\widehat{\xi}_{\tau}=\mathbb{E}\left(\beta_{\tau}^{-1} \beta_{\tau^{\delta}} \xi \mid \mathcal{G}_{\tau-}\right)=\mathbb{E}\left(\bar{\xi}_{\tau} \mid \mathcal{G}_{\tau-}\right) .
$$

In particular, in the no-DVA case with $R=1$, then $\xi=\bar{\xi}=\widehat{\xi}=0$. 
Proposition 4.2 Let there be given semimartingales $\Pi$ and $\Theta$ such that $\Theta=-(Q+\Pi)$ on $\left[0, \bar{\tau}^{\delta}\right]$. The process $\Pi$ is a value process for the member's portfolio if and only if the process $\Theta$ satisfies the following BSDE:

$$
\begin{gathered}
\beta_{t} \Theta_{t}=\mathbb{E}_{t}\left[\sum_{t<\tau_{Z}^{\delta}<\bar{\tau}} \beta_{\tau_{Z}^{\delta}} \epsilon_{\tau_{Z}^{\delta}}-\mathbb{1}_{\{\tau<\bar{T}\}}\left(\beta_{\tau^{\delta}} \xi+\beta_{\tau}(1-\bar{R})\left(P_{\tau-}-\mathcal{C}_{\widehat{\tau}}+\Theta_{\tau-}\right)^{-} J_{t}\right)\right. \\
\left.\quad+\int_{t}^{\bar{\tau}} \beta_{s}\left(g_{s}\left(-P_{s}-\Theta_{s}\right)\right) d s\right], \quad t \in\left[0, \bar{\tau}^{\delta}\right] .
\end{gathered}
$$

Proof. Assuming $\Theta$ defined as $-(Q+\Pi)$ for some value process $\Pi$ on $\left[0, \bar{\tau}^{\delta}\right]$, then the terminal condition $\Theta_{\bar{\tau}^{\delta}}=-\mathbb{1}_{\{\tau<\bar{T}\}} \xi$ that is implicit in 4.9 results from (3.8) and the terminal condition for $\Pi$ in 4.5 . Moreover, we have, for $t \in\left[0, \bar{\tau}^{\delta}\right]$,

$$
-\beta_{t} \Theta_{t}=\beta_{t} Q_{t}+\beta_{t} \Pi_{t}=\beta_{t} P_{t}+\int_{0}^{t} \beta_{s} d D_{s}+\left(\beta_{t} \Pi_{t}-\int_{0}^{t} \beta_{s} J_{s} d D_{s}\right),
$$

hence

$$
\begin{aligned}
& -\beta_{t} \Theta_{t}-\int_{0}^{t} \beta_{s} J_{s}\left(\sum_{Z \subseteq N} \epsilon_{\tau_{Z}^{\delta}} \boldsymbol{\delta}_{\tau_{Z}^{\delta}}(d s)+g_{s}\left(-P_{s}-\Theta_{s}\right) d s\right) \\
& -\mathbb{1}_{\{\tau<\bar{T}\}} \int_{0}^{t}(1-\bar{R})\left(-P_{s-}-\Theta_{s-}+\mathcal{C}_{\widehat{s}}^{\star}\right)^{+} d J_{s}=\left(\beta_{t} P_{t}+\int_{0}^{t} \beta_{s} d D_{s}\right)+\int_{0}^{t} \beta_{s} d \nu_{s},
\end{aligned}
$$

by the pricing BSDE (4.5) satisfied by $\Pi$. In view also of (2.1) (used for $i=0$ here), this is a (local) martingale, hence it coincides with the conditional expectation of its terminal condition (assuming it is a true martingale), which establishes (4.9). The converse implication is proven similarly.

Remark 4.3 As an alternative argument equivalent to the above, one can substitute the right-hand side in (4.6) for $\beta_{t} \Pi_{t}$ in (4.10), which, after an application of the tower rule, yields (4.9). One can proceed similarly to show (4.6) if 4.9 is assumed.

Let, for $\vartheta \in \mathbb{R}$,

$$
\begin{aligned}
\widehat{f}_{t}(\vartheta) & =g_{t}\left(-P_{t}-\vartheta\right)-\gamma_{t} \widehat{\xi}_{t}-(1-\bar{R}) \gamma_{t}\left(P_{t}-\mathcal{C}_{t}^{\star}+\vartheta\right)^{-} \\
& =\underbrace{-\gamma_{t} \widehat{\xi}_{t}}_{d v a_{t}}+\underbrace{\left(c_{t}\left(\mathcal{C}_{t}-P_{\widehat{t}-}\right)+\widetilde{\lambda}_{t}\left(P_{t}-\mathcal{C}_{t}^{\star}+\vartheta\right)^{-}-\lambda_{t}\left(P_{t}-\mathcal{C}_{t}^{\star}+\vartheta\right)^{+}\right)}_{f v a_{t}(\vartheta)},
\end{aligned}
$$

by definition (4.4) of $g$, where $\widetilde{\lambda}=\bar{\lambda}-(1-\bar{R}) \gamma\left(\right.$ recall $\gamma=\gamma^{0}$ is the assumed intensity of $\tau)$. From the perspective of the member, the two terms in the decomposition (4.11) of the coefficient $\widehat{f}_{t}(\vartheta)$ can respectively be interpreted as a beneficial debt valuation adjustment coefficient ( $d v a_{t}$ that can be ignored by setting $R=1$ ) and a funding valuation adjustment coefficient $\left(f v a_{t}(\vartheta)\right.$ in which the DVA2 component can be ignored by setting $\left.\bar{R}=1\right)$.

Proposition 4.3 The "full CCVA BSDE" (4.9) for a semimartingale $\Theta$ on $\left[0, \bar{\tau}^{\delta}\right]$ is equivalent to the following "reduced CCVA BSDE" for a semimartingale $\widehat{\Theta}$ on $[0, \bar{\tau}]$ :

$$
\beta_{t} \widehat{\Theta}_{t}=\mathbb{E}_{t}\left[\sum_{t<\tau_{Z}^{\delta}<\bar{\tau}} \beta_{\tau_{Z}^{\delta}} \epsilon_{\tau_{Z}^{\delta}}+\int_{t}^{\bar{\tau}} \beta_{s} \widehat{f}_{s}\left(\widehat{\Theta}_{s}\right) d s\right], t \in[0, \bar{\tau}],
$$

equivalent in the sense that if $\Theta$ solves $(4.9)$, then $\widehat{\Theta}=J \Theta$ solves $(4.12$, whilst if $\widehat{\Theta}$ solves 4.12 , then $\Theta=J \widehat{\Theta}-(1-J) \mathbb{1}_{\{\tau<\bar{T}\}} \bar{\xi}$ solves 4.9 . 
Proof. The full CCVA BSDE 4.9) is obviously equivalent to $\Theta=-\mathbb{1}_{\{\tau<\bar{T}\}} \bar{\xi}$ on $\left[\bar{\tau}, \bar{\tau}^{\delta}\right]$ and

$$
\begin{aligned}
\beta_{t} \Theta_{t}=\mathbb{E}_{t}\left[\sum_{t<\tau_{Z}^{\delta}<\bar{\tau}} \beta_{\tau_{Z}^{\delta}} \epsilon_{\tau_{Z}^{\delta}}-\mathbb{1}_{\{\tau<\bar{T}\}} \beta_{\tau}\left(\bar{\xi}_{\tau}+(1-\bar{R})\left(P_{\tau-}-\mathcal{C}_{\widehat{\tau}}^{\star}+\Theta_{\tau-}\right)^{-}\right)\right. \\
\left.\quad+\int_{t}^{\bar{\tau}} \beta_{s} g_{s}\left(-P_{s}-\Theta_{s}\right) d s\right]
\end{aligned}
$$

on $[0, \bar{\tau})$, which is in turn equivalent to

$$
\begin{aligned}
& \Theta=-\mathbb{1}_{\{\tau<\bar{T}\}} \bar{\xi} \text { on }\left[\bar{\tau}, \bar{\tau}^{\delta}\right] \text { and, on }[0, \bar{\tau}), \\
& \beta_{t} \Theta_{t}=\mathbb{E}_{t}\left[\sum_{t<\tau_{Z}^{\delta} \leq \bar{\tau}} \beta_{\tau_{Z}^{\delta}} \epsilon_{\tau_{Z}^{\delta}}+\int_{t}^{\bar{\tau}} \beta_{s} \widehat{f}_{s}\left(\Theta_{s}\right) d s\right],
\end{aligned}
$$

because on $[0, \bar{\tau})$ :

$$
\begin{aligned}
\mathbb{E}_{t}[ & \mathbb{1}_{\{\tau<\bar{T}\}} \beta_{\tau}\left(\bar{\xi}_{\tau}+(1-\bar{R})\left(P_{\tau-}-\mathcal{C}_{\widehat{\tau}}^{\star}+\Theta_{\tau-}\right)^{-}\right) \\
& =\mathbb{E}_{t}\left[\mathbb{1}_{\{t<\tau<\bar{T}\}} \beta_{\tau}\left(\widehat{\xi}_{\tau}+(1-\bar{R})\left(P_{\tau-}-\mathcal{C}_{\widehat{\tau}}^{\star}+\Theta_{\tau-}\right)^{-}\right)\right] \\
& =-\mathbb{E}_{t}\left[\int_{t}^{\bar{T}} \beta_{s}\left(\widehat{\xi}_{s}+(1-\bar{R})\left(P_{s-}-\mathcal{C}_{\widehat{s}}^{\star}+\Theta_{s-}\right)^{-}\right) d J s\right] \\
& =\mathbb{E}_{t}\left[\int_{t}^{\bar{T}} \beta_{s} \gamma_{s}\left(\widehat{\xi}_{s}+(1-\bar{R})\left(P_{s-}-\mathcal{C}_{\widehat{s}}^{\star}+\Theta_{s-}\right)^{-}\right) d s\right] .
\end{aligned}
$$

Here the last identity holds by consideration of the (local, assumed true) martingale

$$
\beta_{t}\left(\widehat{\xi}_{t}+(1-\bar{R})\left(P_{\tau-}-\mathcal{C}_{\tau-}^{\star}+\Theta_{\tau-}\right)^{-}\right) d J_{t}+\beta_{t} \gamma_{t}\left(\widehat{\xi}_{t}+(1-\bar{R})\left(P_{t}-\mathcal{C}_{t}^{\star}+\Theta_{t}\right)^{-}\right) d t .
$$

One readily checks that if $\Theta$ solves 4.13 , then $\widehat{\Theta}=J \Theta$ solves 4.12 , whilst if $\widehat{\Theta}$ solves 4.12, then $\Theta=J \widehat{\Theta}-(1-J) \mathbb{1}_{\{\tau<\bar{T}\}} \xi$ solves 4.13.

Note that, provided $r$ and $\tilde{\lambda}$ are bounded fro below, the reduced BSDE coefficient $\widehat{f}_{t}(\vartheta)$ in (4.11) satisfies the monotonicity assumption

$$
\left(\widehat{f}_{t}(\vartheta)-\widehat{f}_{t}\left(\vartheta^{\prime}\right)\right)\left(\vartheta-\vartheta^{\prime}\right) \leq C\left(\vartheta-\vartheta^{\prime}\right)^{2},
$$

for some constant $C$. Then, under mild integrability conditions, the reduced CCVA BSDE (4.12) is well-posed in the space of square integrable solutions (see e.g. Kruse and Popier $(2016$, Sect. 5)). By virtue of Proposition 4.3, so is in turn the full CCVA BSDE (4.9).

Remark 4.4 In the terminology of Crépey and Nguyen (2016), (4.12) is the "partially reduced" CCVA BSDE (cf. also Lemma 2.3 in Crépey and Song (2015)), while the "fully reduced" BSDE (simply called "reduced" in Crépey and Song (2016)) is the BSDE on the time interval $[0, T]$ obtained from 4.12 by projection on a smaller filtration (the market or reference filtration myopic to the defaults of the two parties). In this paper we only work with the partially reduced BSDE in order to avoid the enlargement of filtration technicalities. 


\subsection{Cost of Capital}

The capital at risk of the member is composed of its default fund contribution $D F C_{t}$, which represents implicit capital at risk, and of its regulatory CCP capital $K_{t}^{c m}$ as of (A.3). Along the lines of Albanese et al. (2016), we define the capital valuation adjustment (KVA) of the member as the cost of remunerating its capital at risk $K_{t}=D F C_{t}+K_{t}^{c m}$ at some hurdle rate $k$ throughout the whole life of the portfolio (or until the member defaults). Such a KVA is given by the following formula (cf. Albanese et al. (2016)):

$$
\mathrm{KVA}_{t}=k \mathbb{E}_{t} \int_{t}^{\bar{\tau}} e^{-\int_{t}^{s}\left(r_{u}+k\right) d u} K_{s} d s, t \in[0, \bar{\tau}] .
$$

The KVA-inclusive CCVA is then defined as the sum between our previous CCVA $\Theta$ and this KVA.

\section{Common Shock Model of Default Times}

We use a dynamic Marshall-Olkin (DMO) copula model of the default times $\tau_{i}$ (see Crépey et al. (2014, Chapt. 8-10) and Crépey and Song (2016)). As demonstrated in Crépey et al. (2014, Sect. 8.4), such a model can be efficiently calibrated to marginal and portfolio credit data, e.g. CDS and CDO data (or proxies) on the members. The joint defaults feature of the DMO model is also interesting in regard of the EMIR "cover two" default fund sizing rule (cf. Sect. A.1).

Let there be given a family $\mathcal{Y}$ of "shocks", i.e. subsets $Y$ of members, typically the singletons $\{0\},\{1\}, \ldots,\{n\}$ and a small number of "common shocks" representing simultaneous defaults. For $Y \in \mathcal{Y}$, we define

$$
\eta_{Y}=\inf \left\{t>0 ; \int_{0}^{t} \gamma_{Y}(s) d s>E_{Y}\right\}, \quad J^{Y}=\mathbb{1}_{\left[0, \eta_{Y}\right)},
$$

for a shock intensity function $\gamma_{Y}(t)$ and an independent standard exponential random variable $E_{Y}$. We then set

$$
\tau_{i}=\min _{\{Y \in \mathcal{Y} ; i \in Y\}} \eta_{Y}, \quad i \in N
$$

Example 5.1 Fig. 3 shows one possible default path in a common shock model with $n=5$ and $\mathcal{Y}=\{\{0\},\{1\},\{2\},\{3\},\{4\},\{3,4\},\{1,2,3\},\{0,1\}\}$. The inner ovals show which shocks happen and cause the observed defaults at successive default times. First, the default of name 1 occurs as the consequence of the shock $\{1\}$. Second, names 3 and 4 default simultaneously as a consequence of the shock $\{3,4\}$. Third, the shock $\{1,2,3\}$ triggers the default of name 2 alone (as name 1 and 3 have already defaulted). Fourth, the default of name 0 alone occurs as the consequence of shock $\{0,1\}$. 


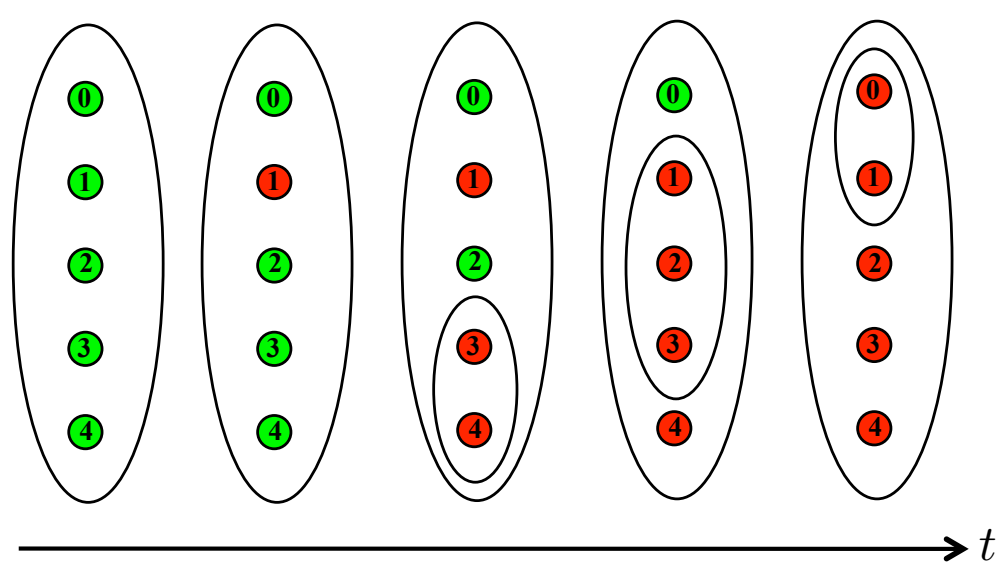

Figure 3: One possible default path in a model with $n=4$ and $\mathcal{Y}=$ $\{\{0\},\{1\},\{2\},\{3\},\{4\},\{3,4\},\{1,2,3\},\{0,1\}\}$.

Again, in the case of the reference member (labeled 0), we omit the superscript 0 in the notation. In particular, we have $J=\mathbb{1}_{[0, \tau)}=\prod_{Y \in \mathcal{Y}_{\bullet}} J^{Y}$, where $\mathcal{Y}_{\bullet}=\{Y \in \mathcal{Y} ; 0 \in Y\}$, hence the intensity $\gamma$ of $\tau$ is given as

$$
\gamma=J_{-} \gamma_{\bullet}, \text { where } \gamma_{\bullet}=\sum_{Y \in \mathcal{Y}_{\bullet}} \gamma_{Y}
$$

We assume that all the market risk factors are gathered in a vector process $\mathbf{X}$ without jump at $\tau$ and that the processes $\mathbf{X}$ and $X=(\mathbf{X}, \mathbf{J})$, where $\mathbf{J}=\left(J^{Y}\right)_{Y \in \mathcal{Y}}$, are Markov in the full model filtration $\mathbb{G}$ given as the filtration of $\mathbf{X}$ progressively enlarged by the random times $\eta_{Y}, Y \in \mathcal{Y}$ (in Sect. 7,8, $\mathrm{X}$ is simply a Black-Scholes stock $S$, augmented by additional factors in order to cope with the potential path dependence of dividends and collateral). Setting $\widehat{\Delta}_{t}=\int_{0}^{t} e^{\int_{s}^{t} r_{u} d u} d D_{s}$ so that $\beta_{t} \Delta_{t}=\beta_{t} \widehat{\Delta}_{t}-\beta_{\tau} \widehat{\Delta}_{\tau-}$ for $t \geq \tau$, we assume, consistent with the interpretation of each respective quantity, that

$$
\begin{aligned}
& \epsilon_{t}=\epsilon\left(t, X_{t}\right) \text { for } t=\tau_{Z}^{\delta}, Z \subseteq N \\
& P_{t}=P\left(t, \mathbf{X}_{t}\right), \widehat{\Delta}_{t}=\widehat{\Delta}\left(t, \mathbf{X}_{t}\right), \mathcal{C}_{t}=\mathcal{C}\left(t, X_{t}\right), \quad t \in[0, \bar{\tau}]
\end{aligned}
$$

(having augmented $\mathbf{X}$ by $\widehat{\Delta}$ and/or $\mathcal{C}$ if need be), for continuous functions $\epsilon(t, x), P(t, \mathbf{x})$, $\widehat{\Delta}(t, \mathbf{x})$ and $\mathcal{C}(t, x)$. In particular, it holds that

$$
\Delta_{\tau}=\widehat{\Delta}_{\tau}-\widehat{\Delta}_{\tau-}=\widehat{\Delta}\left(\tau, \mathbf{X}_{\tau}\right)-\widehat{\Delta}\left(\tau, \mathbf{X}_{\tau-}\right)=0
$$

by continuity of $\mathbf{X}$ at $\tau$ (as opposed to $\Delta_{\tau} \neq 0$ in the gap risk model of Crépey and Song (2016)).

Lemma 5.1 We have

$$
d v a_{t}=d v a\left(t, X_{t}\right)=-J_{t} \bar{\xi}\left(t, X_{t}\right) \gamma_{\bullet}, \mathbb{Q} \times \boldsymbol{\lambda} a . e .,
$$

for a function $\bar{\xi}(t, x)$ such that $\bar{\xi}_{\tau}=\bar{\xi}\left(\tau, X_{\tau-}\right)$. 


\section{$6 \quad$ XVA Engines}

In this section, we summarize in algorithmic terms the central clearing XVA methodology of this paper, as well as a bilateral trading XVA methodology recalled for comparison purposes from Crépey and Song (2016). In both cases we use the common shock model of Sect. 5 for modeling the default times involved.

\subsection{CCVA Engine}

In spite of the nonlinearity inherent to the funding component of the CCVA, standard Monte Carlo loops can be used for estimating a linearized first order CCVA obtained replacing $f v a_{s}\left(\Theta_{s}\right)$ by $f v a_{s}(0)$ in (4.11), i.e. $\widehat{f}_{s}\left(\widehat{\Theta}_{s}\right)$ by $\widehat{f}_{s}(0)$ in 4.12$)$. A nonlinear correction can be estimated based on the Monte Carlo expansion of Fujii and Takahashi (2012a 2012b) (further studied in Gobet and Pagliarani (2015)) in vanilla cases, with explicit formulas for $P_{t}$, or by the branching particles scheme of Henry-Labordère (2012) in more exotic situations. In the bilateral trading setup of Crépey and Song (2016) (see also Crépey and Nguyen (2016)), the nonlinear correction is consistently found less than $5 \%$ to $10 \%$ of the linear part. Hence, in this paper, we just use the linear part. We obtain by first order linear approximation in the reduced CCVA BSDE 4.12):

$$
\begin{aligned}
\Theta_{0}=\widehat{\Theta}_{0} \approx \mathbb{E}\left[\sum_{0<\tau_{Z}^{\delta}<\bar{\tau}} \beta_{\tau_{Z}^{\delta}} \epsilon_{\tau_{Z}^{\delta}}+\int_{0}^{\bar{\tau}} \beta_{s} \widehat{f}_{s}(0) d s\right]=\underbrace{\mathbb{E} \sum_{0<\tau_{Z}^{\delta}<\bar{\tau}} \beta_{\tau_{Z}^{\delta}} \epsilon_{\tau_{Z}^{\delta}}}_{\mathrm{CVA}}+\underbrace{\mathbb{E} \int_{0}^{\bar{\tau}} \beta_{s} d v a_{s} d s}_{\text {DVA }} \\
+\underbrace{\mathbb{E} \int_{0}^{\bar{\tau}} \beta_{s}\left(\widetilde{\lambda}_{s}\left(\mathcal{C}_{s}^{\star}-P_{s}\right)^{+}-\lambda_{s}\left(\mathcal{C}_{s}^{\star}-P_{s}\right)^{-}\right) d s}_{\text {MVA }}+\underbrace{\mathbb{E} \int_{0}^{\bar{\tau}} \beta_{s} c_{s}\left(\mathcal{C}_{s}-P_{\widehat{s}-}\right) d s}_{\text {MLA }},
\end{aligned}
$$

where $\beta_{t}=e^{-\int_{0}^{t} r_{s} d s}, \widetilde{\lambda}=\bar{\lambda}-(1-\bar{R}) \gamma_{\bullet}, \mathcal{C}^{\star}=V M+I M$ and, for each $t=\tau_{Z}^{\delta}<\bar{\tau}$,

$$
\epsilon_{t}=\left(B_{t}-E_{t-}\right)^{+} \frac{D F C_{t}}{\sum_{j \in N} J_{t}^{j} D F C_{t}^{j}}, \text { in which } B_{t}=\sum_{i \in Z}\left(P_{t}^{i}+\Delta_{t}^{i}-\mathcal{C}_{t}^{i}\right)^{+}
$$

with, for each member $i, \mathcal{C}^{i}=V M^{i}+I M^{i}+D F C^{i}$ (cf. (3.11) and (3.7)-(3.9)). In addition, $d v a=-\gamma \widehat{\xi}$, where $\widehat{\xi}$ is a predictable process such that $\xi_{\tau}=\mathbb{E}\left(\beta_{\tau}^{-1} \beta_{\tau^{\delta}} \xi \mid \mathcal{G}_{\tau-}\right)$ (cf. (4.8)), with $\xi=(1-R)\left(P_{\tau^{\delta}}+\Delta_{\tau^{\delta}}-\mathcal{C}_{\tau}\right)^{+}$.

The $\epsilon$ terms in 6.1 give rise to a CVA paid by the member through its contributions to the refill of realized breaches. The terms dubbed MVA and MLA in (6.1), where

$$
\mathcal{C}_{s}^{\star}-P_{s}=P_{\widehat{s}-}+I M_{s}-P_{s} \approx I M_{s} \text { and } \mathcal{C}_{s}-P_{\widehat{s}-}=I M_{s}+D F C_{s},
$$

are interpreted as a margin valuation adjustment (cost to the member of funding its initial margins, essentially) and a margin liquidity adjustment (cost to the member of the CCP margin fees). The positive (respectively negative) terms in (6.1) can be considered as deal adverse (respectively deal friendly) as they increase (respectively decrease) the CCVA $\Theta$. The DVA and the DVA2 can be ignored in $\Theta$ by setting $R=1$ and $\bar{R}=1$, respectively.

For numerical purposes, we use the following randomized version of (6.1):

$$
\mathbb{E}\left[\sum_{0<\tau_{Z}^{\delta}<\bar{\tau}} \beta_{\tau_{Z}^{\delta}} \epsilon_{\tau_{Z}^{\delta}}+\mathbb{1}_{\{\zeta<\bar{\tau}\}} \frac{e^{\mu \zeta}}{\mu} \beta_{\zeta} \widehat{f}_{\zeta}(0)\right]
$$


where $\zeta$ denotes an independent exponential time of parameter $\mu$. Moreover, to deal with the $d v a_{\zeta}$ term in $\widehat{f_{\zeta}}(0)$, we use the following result.

Lemma 6.1 For any predictable process $h$ and independent atomless random variable $\zeta$, we have:

$$
\mathbb{E}\left[\mathbb{1}_{\{\zeta<\bar{\tau}\}} h_{\zeta} \beta_{\zeta} d v a\left(\zeta, X_{\zeta}\right)\right]=-\mathbb{E}\left[\mathbb{1}_{\{\zeta<\bar{\tau}\}} h_{\zeta} \beta_{\zeta+\delta}(1-R) \gamma_{\bullet}(\zeta)\left(Q_{\zeta^{\delta}}-\mathcal{C}_{\zeta}^{\star}\right)^{+}\right] .
$$

Plugging $h_{\zeta}=\frac{e^{\mu \zeta}}{\mu}$ in 6.3 to deal with the $d v a_{\zeta}$ term in $\widehat{f}_{\zeta}(0), 6.2$ is rewritten as

$$
\begin{aligned}
& \widehat{\Theta}_{0} \approx \mathbb{E}\left\{\sum_{0<\tau_{Z}^{\delta}<\bar{\tau}} \beta_{\tau_{Z}^{\delta}} \epsilon_{\tau_{Z}^{\delta}}+\mathbb{1}_{\{\zeta<\bar{\tau}\}} \frac{e^{\mu \zeta}}{\mu} \times\right. \\
& \left.\left[-\beta_{\zeta^{\delta}} \gamma_{\bullet}(\zeta)(1-R)\left(Q_{\zeta^{\delta}}-\mathcal{C}_{\zeta}\right)^{+}+\beta_{\zeta}\left(\widetilde{\lambda}_{\zeta}\left(\mathcal{C}_{\zeta}^{\star}-P_{\zeta}\right)^{+}-\lambda_{\zeta}\left(\mathcal{C}_{\zeta}^{\star}-P_{\zeta}\right)^{-}\right)\right]\right\} .
\end{aligned}
$$

The KVA-inclusive CCVA is then defined as the sum between 6.4 and a KVA as of 4.14, valued at time $t=0$ by simulation and randomization of the time integral there.

\subsection{BVA Engine}

Here we provide an executive summary of a bilateral CSA trading setup recalled for comparison purposes from Crépey and Song (2016) (cf. also Brigo and Pallavicini (2014) or Bichuch, Capponi, and Sturm (2016) for related bilateral counterparty risk analyses with asymmetric funding costs).

Remark 6.1 In Crépey and Song (2016), the cash flows are viewed from the perspective of the bank, which will be taken as the reference member here, whereas we view them in this paper from the perspective of the clearing house, i.e. opposite to the one of the member. Hence, the sign conventions are opposite, i.e. $P, \Delta, Q$, etc... in this paper correspond to their opposites in Crépey and Song (2016), which is why we see ${ }^{\mp}$ here whenever we have . \pm there.

In the context of bilateral trading between a bank, taken as the reference member labeled by 0 in the previous CCP setup, and a counterparty taken as another member $i \neq 0$, let $V M$ denote the variation margin, where $V M \geq 0$ (resp. $\leq 0$ ) means collateral posted by the bank and received by the counterparty (resp. posted by the counterparty and received by the bank). Let $I^{b} \geq 0$ and $I^{c} \leq 0$ represent the initial margin posted by the bank and the negative of the initial margin posted by the counterparty. Hence,

$$
C^{b}=V M+I^{b} \text { and } C^{c}=V M+I^{c}
$$

are the total collateral guarantee for the counterparty and the negative of the total collateral guarantee for the bank. Assuming the variation margins re-hypothecable and the initial margins segregated (as typically so in practice), the collateral funded by the bank is $C=$ $V M+I^{b}$. For consistency with our CCP setup, $V M_{t}$ will be taken as $P_{\widehat{t}-}$. So, in the spirit of a standard CSA, we are considering full collateralization, and even overcollateralization through the initial margins. We assume that $V M$ and $I^{b}$ are remunerated at the OIS rate $r$. Following Crépey and Song (2016), at time 0, the difference $\Theta_{0}$ between the markto-market of the portfolio and its value inclusive of counterparty and funding risk (both 
from the perspective of the bank, cf. the remark 4.2), difference dubbed BVA for bilateral valuation adjustment, can be linearized as follows:

$$
\begin{aligned}
\Theta_{0}=\bar{\Theta}_{0} \approx \mathbb{E}\left[\int_{0}^{\bar{\tau}} \beta_{s} \bar{f}_{s}(0) d s\right]=\underbrace{\mathbb{E} \int_{0}^{\bar{\tau}} \beta_{s} c d v a_{s} d s}_{\mathrm{CDVA}}+ \\
+\underbrace{\mathbb{E} \int_{0}^{\bar{\tau}} \beta_{s}\left(\tilde{\lambda}_{s}\left(C_{s}-P_{s}\right)^{+}-\lambda_{s}\left(C_{s}-P_{s}\right)^{-}\right) d s}_{\text {MVA }} .
\end{aligned}
$$

Here:

- $P$ means the mark-to-market of the position of the member with the counterparty (viewed from the perspective of the latter),

- the meaning of $\beta, \widetilde{\lambda}$ and $\lambda$ is as in the CCP setup,

- $\tau=\tau_{b} \wedge \tau_{c}$ is the first-to-default time of the bank and the counterparty,

- $c d v a=\gamma \widehat{\xi}$, where $\widehat{\xi}$ is a predictable process such that $\widehat{\xi}_{\tau}=\mathbb{E}\left(\beta_{\tau}^{-1} \beta_{\tau^{\delta}} \xi \mid \mathcal{G}_{\tau-}\right)$, with

$$
\xi=\mathbb{1}_{\left\{\tau_{c} \leq \tau_{b}^{\delta}\right\}}\left(1-R_{c}\right)\left(P_{\tau^{\delta}}+\Delta_{\tau^{\delta}}-C_{\tau}^{c}\right)^{-}-\mathbb{1}_{\left\{\tau_{b} \leq \tau_{c}^{\delta}\right\}}\left(1-R_{b}\right)\left(P_{\tau^{\delta}}+\Delta_{\tau^{\delta}}-C_{\tau}^{b}\right)^{+},
$$

in which the recovery rates $R_{c}$ of the counterparty to the bank and $R_{b}$ of the bank to the counterparty are usually taken in a bilateral trading setup as $40 \%$.

For numerical purposes, we use the following randomized version of 6.6$)$ :

$$
\mathbb{E}\left[\mathbb{1}_{\{\zeta<\bar{\tau}\}} \frac{e^{\mu \zeta}}{\mu} \beta_{\zeta} \bar{f}_{\zeta}(0)\right]
$$

where $\zeta$ denotes an independent exponential time of parameter $\mu$. The $c d v a_{\zeta}$ term in $\bar{f}_{\zeta}(0)$ is treated by the following bilateral analog of Lemma 6.1. We write $\mathcal{Y}_{b}=\{Y \in \mathcal{Y} ; 0 \in Y\}$, $\mathcal{Y}_{c}=\{Y \in \mathcal{Y} ; i \in Y\}$ and we recall that $X=(\mathbf{X}, \mathbf{J})$ denotes the market risk and common shock factor process introduced in Sect. 5, assumed without jump at $\tau$. Similar to Lemma 5.1. it holds that $c d v a_{t}=c d v a\left(t, X_{t}\right)$. In addition (see Lemma 8.2 and its proof in Crépey and Song (2016, hal version 2), in a slightly more general setup where $\mathbf{X}$ may jump at $\tau$ ):

Lemma 6.2 For any predictable process $h$ and independent atomless random variable $\zeta$, we have:

$$
\begin{aligned}
& \mathbb{E}\left[\mathbb{1}_{\{\zeta<\bar{\tau}\}} h_{\zeta} \beta_{\zeta} \operatorname{dva}\left(\zeta, X_{\zeta}\right)\right]=\mathbb{E}\left[\mathbb{1}_{\{\zeta<\bar{\tau}\}} h_{\zeta} \beta_{\zeta^{\delta}} \times\right. \\
& \left(\left(\sum_{Y \in \mathcal{Y}_{c}} \gamma_{Y}(\zeta)+\mathbb{1}_{\left\{\tau_{c} \leq \zeta^{\delta}\right\}} \sum_{Y \in \mathcal{Y}_{b} \backslash \mathcal{Y}_{c}} \gamma_{Y}(\zeta)\right)\left(1-R_{c}\right)\left(Q_{\zeta^{\delta}}-C_{\zeta}\right)^{-}\right. \\
& \left.\left.\quad-\left(\sum_{Y \in \mathcal{Y}_{b}} \gamma_{Y}(\zeta)+\mathbb{1}_{\left\{\tau_{b} \leq \zeta^{\delta}\right\}} \sum_{Y \in \mathcal{Y}_{c} \backslash \mathcal{Y}_{b}} \gamma_{Y}(\zeta)\right)\left(1-R_{b}\right)\left(Q_{\zeta^{\delta}}-C_{\zeta}^{b}\right)^{+}\right)\right] .
\end{aligned}
$$


Plugging $h_{\zeta}=\frac{e^{\mu \zeta}}{\mu}$ in 6.8 to deal with the $c d v a_{\zeta}$ term in $\bar{f}_{\zeta}(0)$, 6.7) is rewritten as (compare (6.4)):

$$
\begin{aligned}
\bar{\Theta}_{0} \approx & \mathbb{E}\left\{\mathbb { 1 } _ { \{ \zeta < \overline { \tau } \} } \frac { e ^ { \mu \zeta } } { \mu } \left[\beta _ { \zeta ^ { \delta } } \left(\left(\sum_{Y \in \mathcal{Y}_{c}} \gamma_{Y}(\zeta)+\mathbb{1}_{\left\{\tau_{c} \leq \zeta^{\delta}\right\}} \sum_{Y \in \mathcal{Y}_{b} \backslash \mathcal{Y}_{c}} \gamma_{Y}(\zeta)\right)\left(1-R_{c}\right)\left(Q_{\zeta^{\delta}}-C_{\zeta}\right)^{-}\right.\right.\right. \\
& \left.-\left(\sum_{Y \in \mathcal{Y}_{b}} \gamma_{Y}(\zeta)+\mathbb{1}_{\left\{\tau_{b} \leq \zeta^{\delta}\right\}} \sum_{Y \in \mathcal{Y}_{c} \backslash \mathcal{Y}_{b}} \gamma_{Y}(\zeta)\right)\left(1-R_{b}\right)\left(Q_{\zeta^{\delta}}-C_{\zeta}^{b}\right)^{+}\right) \\
+ & \left.\left.\beta_{\zeta}\left(\widetilde{\lambda}_{\zeta}\left(P_{\zeta}-\mathcal{C}_{\zeta}\right)^{-}-\lambda_{\zeta}\left(P_{\zeta}-\mathcal{C}_{\zeta}\right)^{+}\right)\right]\right\}
\end{aligned}
$$

Such adjustments are then computed counterparty by counterparty and added over $i=$ $1, \ldots, n$ to obtain the BVA of the bank.

Remark 6.2 In practice, netting sets typically merge into a unique funding set, meaning that one should solve for a single MVA at the level of the whole portfolio of the bank. However, in the present frictionless variation-margining case (cf. the remark 3.1),

$$
\mathcal{C}_{\zeta}-P_{\zeta}=P_{\widehat{\zeta}-}+I M_{\zeta}-P_{\zeta} \approx I M_{\zeta} \geq 0
$$

holds counterparty by counterparty, so that a unique funding set or funding by netting sets makes a negligible difference in practice.

Similar as in the CCP setup, the KVA-inclusive BVA is obtained by adding to (6.9) a KVA in the sense of the formula (4.14) (valued at $t=0$ ), except that $K$ is now the bilateral regulatory capital given by the formulas of Sect. A.2.

\section{Experimental Framework}

In this section we design an experimental framework that is used for the XVA comparative numerical analysis of Sect. 8 .

\subsection{Driving Asset}

Given an interest rate process $S$, we consider a stylized swap of strike $\bar{S}$ with cash flows $h_{l}\left(\bar{S}-S_{T_{l-1}}\right)$ at increasing times $T_{l}, l=1, \ldots, d$, where $h_{l}=T_{l}-T_{l-1}$. We suppose a stylized Black-Scholes dynamics with risk-neutral drift $\kappa$ and volatility $\sigma$ for the interest rate process $S$. Denoting by $T_{l_{t}}$ the smallest $T_{l}>t$, the mark-to-market of the swap for the party receiving the above cash flows is given, for $T_{0}=0 \leq t \leq T_{d}=\bar{T}$, by $P_{t}=\beta_{t}^{-1} \beta_{T_{l_{t}}} h_{l}\left(\bar{S}-S_{T_{l_{t}-1}}\right)+P_{t}^{\star}$, where

$$
P_{t}^{\star}=\beta_{t}^{-1} \bar{S} \sum_{l=l_{t}+1}^{d} \beta_{T_{l}} h_{l}-\beta_{t}^{-1} S_{t} \sum_{l=l_{t}+1}^{d} \beta_{T_{l}} h_{l} e^{\kappa\left(T_{l-1}-t\right)}=P_{\star}\left(t, S_{t}\right) .
$$

We choose the notional Nom of the swap and its strike $\bar{S}$ in such a way that each leg of the swap has a mark-to-market equal to one at time 0. 


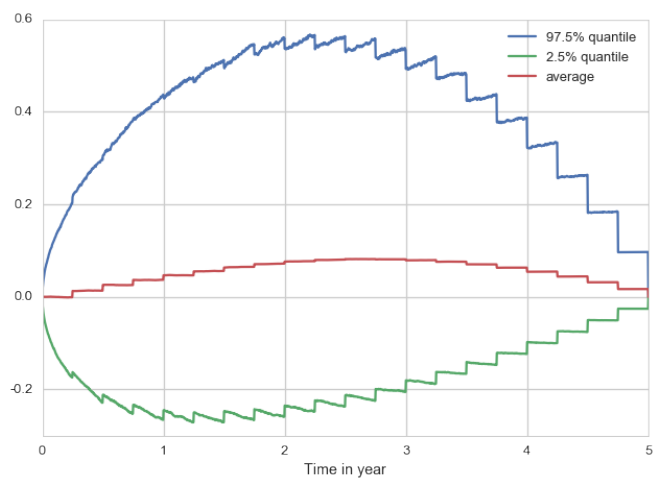

Figure 4: Mark-to-market process of the swap viewed from the point of view of a party receiving floating and paying fix in the swap (party with a long unit position in the swap). The mean and quantiles as a function of time are computed by Monte Carlo simulation of the process $\left(-P_{t}\right)$ based on the formula (7.1) for $P_{\star}$, used along $m=10^{4}$ simulated trajectories of $S$.

The following numerical values are used:

$$
r=2 \%, \quad S_{0}=100, \quad \kappa=12 \%, \sigma=20 \%, \quad h_{l}=3 \text { months, } \bar{T}=5 \text { years, }
$$

resulting in the mark-to-market process displayed in Figure 4 from the point of view of a party receiving floating and paying fix, which we call a long unit position in the swap. Figure 4 exhibits the typical profile of an interest rate swap in an increasing term structure of interest rates, where expectations of increasing rates make the swap in the money on average (i.e. the average curve is in the positive in Figure 4). This yields to the product the XVA flavor that would be absent in a flat interest rates environment where the mark-tomarket process of the swap would be zero and not give rise to any adjustments. The present Black-Scholes setup and values of the parameters for the process $S$ allow us to obtain this stylized pattern without having to introduce a full flesh interest rate model, which would add useless complexity with respect to our goal in this paper.

\subsection{Structure of the Clearing house}

We consider a clearing house with $(n+1)$ members chosen among the 125 names of the CDX index as of 17 December 2007, a particular day toward the beginning of the global financial crisis. The default times of the 125 names are modeled by a common shock model with piecewise constant intensities $\gamma_{Y}$ constant on the time intervals $[0,3]$ and $[3,5]$ years, calibrated to the corresponding 3 and 5 year CDS and 5 year CDO data. With five nested common shocks $Y$ on top of an idiosyncratic shock $Y=\{i\}$ for each of the 125 names, a nearly perfect calibration can be achieved, as developed in Crépey et al. (2014, Sect. 8.4.3)

We consider a subset of nine representative members of the index, with increasing CDS spreads shown in the first row of Table 1 . 


\begin{tabular}{|c|c|c|c|c|c|c|c|c|c|}
\hline$\Sigma_{i}$ & 45 & 52 & 56 & 61 & 73 & 108 & 176 & 367 & 1053 \\
\hline$\alpha_{i}$ & $(0.46)$ & 0.09 & 0.23 & $(0.05)$ & 0.34 & $(0.04)$ & 0.69 & $(0.44)$ & $(0.36)$ \\
\hline
\end{tabular}

Table 1: (Top) Average 3 and 5 year CDS spreads $\Sigma_{i}$, in basis points (bp), for a representative subset of nine members of the CDX index as of 17 December 2007. (Bottom) Coefficients $\alpha_{i}$ summing up to 0 used for determining the swap positions of the nine members.

The coefficients $\alpha_{i}$ in the second row, where parentheses mean negative numbers, will be used in a way explained below for determining the positions in the swap of the nine members in the simulations. These coefficients were obtained as the difference between a vector of nine uniform numbers and its cyclic shift, so that $\sum_{i \in N} \alpha_{i}=0$.

\subsection{Member Portfolios}

We represent in an antisymmetric matrix form

$$
\varpi=\begin{gathered}
0 \\
0 \\
1 \\
2 \\
3 \\
3 \\
\vdots \\
n
\end{gathered}\left(\begin{array}{cccccc}
0 & \varpi_{0,1} & \varpi_{0,2} & \varpi_{0,3} & \cdots & \varpi_{0, n} \\
\cdot & 0 & \varpi_{1,2} & \varpi_{1,3} & \cdots & \varpi_{1, n} \\
. & . & 0 & \varpi_{2,3} & \cdots & \varpi_{2, n} \\
. & . & . & 0 & \cdots & \varpi_{3, n} \\
\vdots & \vdots & \vdots & \vdots & \ddots & \vdots \\
. & . & . & . & \cdots & 0
\end{array}\right),
$$

where each "." represents the negative of the symmetric entry in the matrix, the positions of each member $i$ with respect to each member $j$ (or short positions of $j$ with respect to i) in the swap. Note that the data of the CCVA BSDE related to the member 0, or of the linearized time-0 CCVA formula (6.4), only depend on the matrix $\varpi$ through the sums of each of its rows, corresponding to the vector of the short positions of the different clearing members against the CCP. By contrast, the data of the BVA BSDE related to the member 0 , or of the linearized time-0 BVA formula $(6.9)$, only depend on the matrix $\varpi$ through its first row (vector of the short positions of the different counterparties $i=1, \ldots, n$ against the reference member 0 ). Hence, we can forget about the detail of the above matrix, focusing on the $\omega_{i}^{c s a}:=\varpi_{0, i}$ and $\omega_{i}^{c c p}:=\sum_{l \neq i} \varpi_{l, i}, i \neq 0$, for comparing two trading setups:

- A CSA setup as of Sect. 6.2, where each member $i \neq 0$ trades a short $\omega_{i}^{\text {csa }} \in \mathbb{R}$ position in the swap with the member 0 , whichever other trades members $i \neq 0$ may have between each others.

- For instance, but non necessarily, each member $i \neq 0$ has a short $\omega_{i}^{\text {csa }} \in \mathbb{R}$ position with the member 0 and there are no other trades between members (at least after netting at the level of each pair of members), which corresponds to the situation where only the first row and column are nonzero in the matrix $\varpi$.

- In any case, the netted long position of the member 0 is $\sum_{i \neq 0} \omega_{i}^{c s a}$. However, netting does not apply across different counterparties in the CSA setup. We call compression factor $\nu_{0}$ the gross position of the reference member 0 , i.e. the 
number $\nu_{0}=\sum_{i \neq 0}\left|\omega_{i}^{c s a}\right|$ of trades the member 0 is engaged into in the CSA setup.

- A CCP setup as of Sect. 6.1. where each member $i \neq 0$ trades a short $\omega_{i}^{c c p} \in \mathbb{R}$ position in the swap through the CCP $\left(\omega_{i}^{c c p} \leq 0\right.$ effectively means a long position of member $i$ ), whichever way this position may be distributed among other members.

- For instance, but non necessarily, each member $i \neq 0$ has a short $\omega_{i}^{c c p} \in \mathbb{R}$ position with the member 0 and there are no other trades between members, which again corresponds to the situation where only the first row and column are nonzero in $\varpi$.

- In any case, since members trade between themselves, the member has a $\sum_{i \neq 0} \omega_{i}^{c c p}$ position in the driving asset after netting through the CCP, instead of a non netted position of size $\nu_{0}$ before clearing through the CCP.

Moreover, in order to obtain diverse while comparable setups, we will alternately consider as reference member 0 each of the nine members in Table 1, for positions in the driving asset determined by the coefficients $\alpha_{i}$ (summing up to zero) in the second row of Table 1 through the following rule: $\omega_{i}=-\frac{\alpha_{i}}{\alpha_{0}}, i \neq 1$ (where $\omega=\omega^{c s a}$ or $\omega^{c c p}$, as suitable). Since the coefficients $\alpha_{i}$ add up to 0 , this specification ensures $\sum_{i \neq 0} \omega_{i}=1$, i.e. a netted position of the member 0 (whoever it is), always equal to 1 in the CCP setup. We also define $\omega_{0}=-\frac{\alpha_{0}}{\alpha_{0}}=-1$, consistent with the member 0 being long a +1 , i.e. short $\mathrm{a}-1$, net position in the swap in the CCP setup (in the CSA setup this value of $\omega_{0}$ is purely conventional).

Note that

$$
\nu_{0}=\sum_{i \neq 0}\left|\omega_{i}\right|=\sum_{i \neq 0} \frac{\left|\alpha_{i}\right|}{\left|\alpha_{0}\right|}=\frac{\sum_{i \in N}\left|\alpha_{i}\right|}{\left|\alpha_{0}\right|}-1,
$$

so the smaller $\left|\alpha_{0}\right|$, the larger the compression factor $\nu_{0}$ (gross position of the reference member when trading bilaterally, whereas its net, centrally cleared position is equal to one).

Example 7.1 Table 2 shows the resulting values of the $\omega_{i}$ of the different members $i \neq 0$ when the name with CDS spread $61 \mathrm{bp}$ (name with the second smallest $\left|\alpha_{i}\right|$ in Table 1 . with corresponding entries emphasized in bold in Table 2 is taken as reference member 0 (prototype of a name with a large gross position). Hence, the $\omega_{i}$ in Table 2 are proportional to the $\alpha_{i}$ in Table 1. modulo a scaling factor so that the $\omega_{i}$ of this particular name (then labeled as 0 ) is -1 . In this case $\nu_{0}=\sum_{i \neq 0}\left|\omega_{i}\right|=53.00$.

\begin{tabular}{|c|c|c|c|c|c|c|c|c|c|}
\hline$\Sigma$ & 45 & 52 & 56 & $\mathbf{6 1}$ & 73 & 108 & 176 & 367 & 1053 \\
\hline$\omega$ & $(9.20)$ & 1.80 & 4.60 & $\mathbf{( 1 . 0 0 )}$ & 6.80 & $(0.80)$ & 13.80 & $(8.80)$ & $(7.20)$ \\
\hline
\end{tabular}

Table 2: Positions $\omega_{i}$ in the swap of the nine members with CDS spreads $\Sigma_{i}$, in the respective $\omega_{i}=\omega_{i}^{c s a}$ or $\omega_{i}^{c c p}$ meaning, when the reference member 0 is the name with CDS spread 61 bp and the second smallest $\left|\alpha_{i}\right|$ in Table 1 .

Example 7.2 Table 3 is the analog of Table 2 when the member with spread 367 bp (name with the second largest credit spread in Table 1, with corresponding entries emphasized in 
bold in Table 2) is taken as reference member 0 (prototype of a risky name). In this case $\nu_{0}=\sum_{i \neq 0}\left|\omega_{i}\right|=5.14$.

\begin{tabular}{|c|c|c|c|c|c|c|c|c|c|}
\hline$\Sigma_{i}$ & 45 & 52 & 56 & 61 & 73 & 108 & 176 & $\mathbf{3 6 7}$ & 1053 \\
\hline$\omega_{i}$ & $(1.05)$ & 0.20 & 0.52 & $(0.11)$ & 0.77 & $(0.09)$ & 1.57 & $\mathbf{( 1 . 0 0 )}$ & $(0.82)$ \\
\hline
\end{tabular}

Table 3: Analog of Table 2 when the reference member 0 is the name with CDS spread 367 bp (name with the second largest credit spread $\Sigma_{i}$ ) in Table 1

\subsection{Margins}

CCP setup The initial margin $I M^{i}$ posted by each member $i \in N$ is set through (3.4), using as risk measure $\rho$ the risk-neutral value at risk of some level $a$ "close to 1 " . Since the pricing function $P_{\star}$ in $(7.1)$ is decreasing in $S$, therefore $I M^{i}$ can be proxied, at each simulated time $\zeta$ in 6.4 or $(6.9)$, by

$$
I M_{\zeta}^{i}=N o m \times\left|\omega_{i}\right| \times \begin{cases}P_{\star}\left(\zeta, S_{\zeta}\right)-P_{\star}\left(\zeta, S_{\zeta} e^{\sigma \sqrt{\delta^{\prime}} \Phi^{-1}(a)+\left(\kappa-\frac{\sigma^{2}}{2}\right) \delta^{\prime}}\right), & \omega_{i} \geq 0 \\ P_{\star}\left(\zeta, S_{\zeta} e^{\sigma \sqrt{\delta^{\prime}} \Phi^{-1}(1-a)+\left(\kappa-\frac{\sigma^{2}}{2}\right) \delta^{\prime}}\right)-P_{\star}\left(\zeta, S_{\zeta}\right), & \omega_{i} \leq 0,\end{cases}
$$

where $\Phi$ is the standard normal cdf and where we recall that $\delta^{\prime}=\delta+h$ is the margin period of risk.

For instance, the reference member 0 , with $\omega_{0}^{c c p}=-1$, is long one unit in the swap with mark-to-market profile shown in Figure 4, hence the exposure of the CCP to member 0 is the opposite profile. Accordingly (recalling that Figure 4 shows $\left(-P_{t}\right)$ ), the CCP asks initial margins to the member 0 based on $P_{\star}\left(\zeta, S_{\zeta} e^{\sigma \sqrt{\delta^{\prime}} \Phi^{-1}(1-a)+\left(\mu-\frac{\sigma^{2}}{2}\right) \delta^{\prime}}\right)-$ $P_{\star}\left(\zeta, S_{\zeta}\right)$, consistent with the second line in $(7.2)$ in case $\omega_{i=0} \leq 0$.

Consistently with a "cover two" EMIR rule (see Sect. A.1), the default fund contributions are set as the sum of the two largest exposures of the clearing members (exposures in the sense of their EADs as explained in Sect. 7.5), allocated between them proportionally to their initial margins.

CSA setup The initial margin $-I^{c} \geq 0$ required by the member 0 from the member $i \neq 0$ (cf. (6.5) ) is given by the right-hand side formula in (7.2) valued at some quantile level $a$ (possibly different from the one used in the CCP setup).

For instance, if $\omega_{i}^{c s a}=+2$, meaning that the member 0 has a "double Figure 4 exposure" with regard to counterparty $i$, then the member 0 asks the counterparty $i$ to post initial margins based on $P_{\star}\left(\zeta, S_{\zeta}\right)-P_{\star}\left(\zeta, S_{\zeta} e^{\sigma \sqrt{\delta^{\prime}} \Phi^{-1}(a)+\left(\kappa-\frac{\sigma^{2}}{2}\right) \delta^{\prime}}\right)$ (recall again that Figure 4 shows $\left.\left(-P_{t}\right)\right)$, consistent with the use of the first branch in $(7.2)$ in the case where $\omega_{i}^{c s a} \geq 0$ (for $\left.i \neq 0\right)$.

Symmetrically, the formula for the initial margin $I^{b} \geq 0$ required by the member $i$ from the member 0 reads

$$
I_{\zeta}^{b}=-\omega_{i} \times N o m \times \begin{cases}P_{\star}\left(\zeta, S_{\zeta}\right)-P_{\star}\left(\zeta, S_{\zeta} e^{\sigma \sqrt{\delta^{\prime}} \Phi^{-1}(a)+\left(\kappa-\frac{\sigma^{2}}{2}\right) \delta^{\prime}}\right), & \omega_{i} \leq 0 \\ P_{\star}\left(\zeta, S_{\zeta}\right)-P_{\star}\left(\zeta, S_{\zeta} e^{\sigma \sqrt{\delta^{\prime}} \Phi^{-1}(1-a)+\left(\kappa-\frac{\sigma^{2}}{2}\right) \delta^{\prime}}\right), & \omega_{i} \geq 0 .\end{cases}
$$




\subsection{Exposure-at-defaults}

The prime motivation for the Black-Scholes model used for $S$ and for our risk-neutral valueat-risk for the IMs is that these give rise to an explicit formula for the exposure-at-defaults (EAD), which are the basic primitive of all the regulatory capital formulas. This avoids the computational burden of nested Monte Carlo simulations (see the introductory paragraph to Sect. A). We also use EADs as a proxy of the exposures of the members in the context of our EMIR "cover two" default fund computations (cf. Sect. A.1).

In fact, for any grid time $v=t+\epsilon p$ involved in EAD computations (cf. (A.2), (3.2) and (7.1), with $\epsilon$ taken as one month in the numerics), we have in our model:

$$
\begin{aligned}
& \mathbb{E}_{t}\left[\left(P_{v+\delta^{\prime}}+\int_{\left[v, v+\delta^{\prime}\right]} e^{\int_{s}^{v+\delta^{\prime}} r_{u} d u} d D_{s}-P_{v-}-I M_{v}\right)^{+}\right] \\
& =\mathbb{E}_{t}\left[\left(P^{\star}\left(v+\delta^{\prime}, S_{v+\delta^{\prime}}\right)-P^{\star}\left(v, S_{v}\right)-\mathbb{V} a \mathbb{R}_{t}\left(P^{\star}\left(v+\delta^{\prime}, S_{v+\delta^{\prime}}\right)-P^{\star}\left(v, S_{v}\right)\right)^{+}\right]\right. \\
& =\mathbb{E}_{t} \mathbb{E}_{v}\left[\left(P^{\star}\left(v+\delta^{\prime}, S_{v+\delta^{\prime}}\right)-P^{\star}\left(v, S_{v}\right)-\mathbb{V} a \mathbb{R}_{t}\left(P^{\star}\left(v+\delta^{\prime}, S_{v+\delta^{\prime}}\right)-P^{\star}\left(v, S_{v}\right)\right)^{+}\right],\right.
\end{aligned}
$$

where $\mathbb{V} a \mathbb{R}$ represents the risk-neutral value-at-risk of level $a$. Denoting by $\mathbb{E} \mathbb{S}$ the corresponding expected shortfall, the conditional version of the identity $\mathbb{E}\left[X \mathbb{1}_{X \geq \mathbb{V} a \mathbb{R}(X)}\right]=$ $(1-a) \mathbb{E} \mathbb{S}(X)$ yields

$$
\begin{aligned}
& \mathbb{E}_{v}\left[\left(P^{\star}\left(v+\delta^{\prime}, S_{v+\delta^{\prime}}\right)-P^{\star}\left(v, S_{v}\right)-\mathbb{V} a \mathbb{R}_{t}\left(P^{\star}\left(v+\delta^{\prime}, S_{v+\delta^{\prime}}\right)-P^{\star}\left(v, S_{v}\right)\right)^{+}\right]\right. \\
& =(1-a)\left(\mathbb{E}_{v}\left(P^{\star}\left(v+\delta^{\prime}, S_{v+\delta^{\prime}}\right)-P^{\star}\left(v, S_{v}\right)\right)-\mathbb{V} a \mathbb{R}_{v}\left(P^{\star}\left(v+\delta^{\prime}, S_{v+\delta^{\prime}}\right)-P^{\star}\left(v, S_{v}\right)\right)\right) \\
& =(1-a)\left(e^{\sigma \sqrt{\delta^{\prime}} \Phi^{-1}(a)}-e^{\sigma \sqrt{\delta^{\prime}} \frac{\phi\left(\Phi^{-1}(a)\right)}{a}}\right) \beta_{v+\delta^{\prime}}^{-1} e^{-\kappa v-\frac{\sigma^{2}}{2} \delta^{\prime}} S_{v} \sum_{l=l_{v+\delta^{\prime}}}^{d} \beta_{T_{l}} h_{l} e^{\kappa T_{l-1}},
\end{aligned}
$$

where $\Phi$ and $\phi$ are the standard normal cdf and density. Hence,

$$
\mathbb{E}_{t}\left[\left(P_{v+\delta^{\prime}}+\int_{\left[v, v+\delta^{\prime}\right]} e^{\int_{s}^{v+\delta^{\prime}} r_{u} d u} d D_{s}-P_{v-}-I M_{v}\right)^{+}\right]=f_{v}^{a, \delta^{\prime}} \times(1-a) e^{-\kappa t} S_{t},
$$

where

$$
f_{v}^{a, \delta^{\prime}}=\left(e^{\sigma \sqrt{\delta^{\prime}} \Phi^{-1}(a)}-e^{\sigma \sqrt{\delta^{\prime}} \frac{\phi\left(\Phi^{-1}(a)\right)}{1-a}}\right) \beta_{v+\delta^{\prime}}^{-1} e^{-\frac{\sigma^{2}}{2} \delta^{\prime}} \sum_{l=l_{v+\delta^{\prime}}}^{d} \beta_{T_{l}} h_{l} e^{\kappa T_{l-1}} .
$$

Likewise, we have

$$
\mathbb{E}_{t}\left[\left(P_{v+\delta^{\prime}}+\int_{\left[v, v+\delta^{\prime}\right]} e^{\int_{s}^{v+\delta^{\prime}} r_{u} d u} d D_{s}-P_{v-}-I M_{v}\right)^{-}\right]=g_{v}^{a, \delta^{\prime}} \times(1-a) e^{-\kappa t} S_{t},
$$

where

$$
g_{v}^{a, \delta^{\prime}}=-\left(e^{\sigma \sqrt{\delta^{\prime}} \Phi^{-1}(1-a)}-e^{-\sigma \sqrt{\delta^{\prime}} \frac{\phi\left(\Phi^{-1}(a)\right)}{1-a}}\right) \beta_{v+\delta^{\prime}}^{-1} e^{-\frac{\sigma^{2}}{2} \delta^{\prime}} \sum_{l=l_{v+\delta^{\prime}}}^{d} \beta_{T_{l}} h_{l} e^{\kappa T_{l-1}} .
$$

Based on (7.3) through (7.4), explicit formulas for the EADs follows. Figure 5 shows the time-0 EADs of the nine CCP members for their positions in the swap corresponding to the choice of the name of examples 7.1 or 7.2 as reference member. 

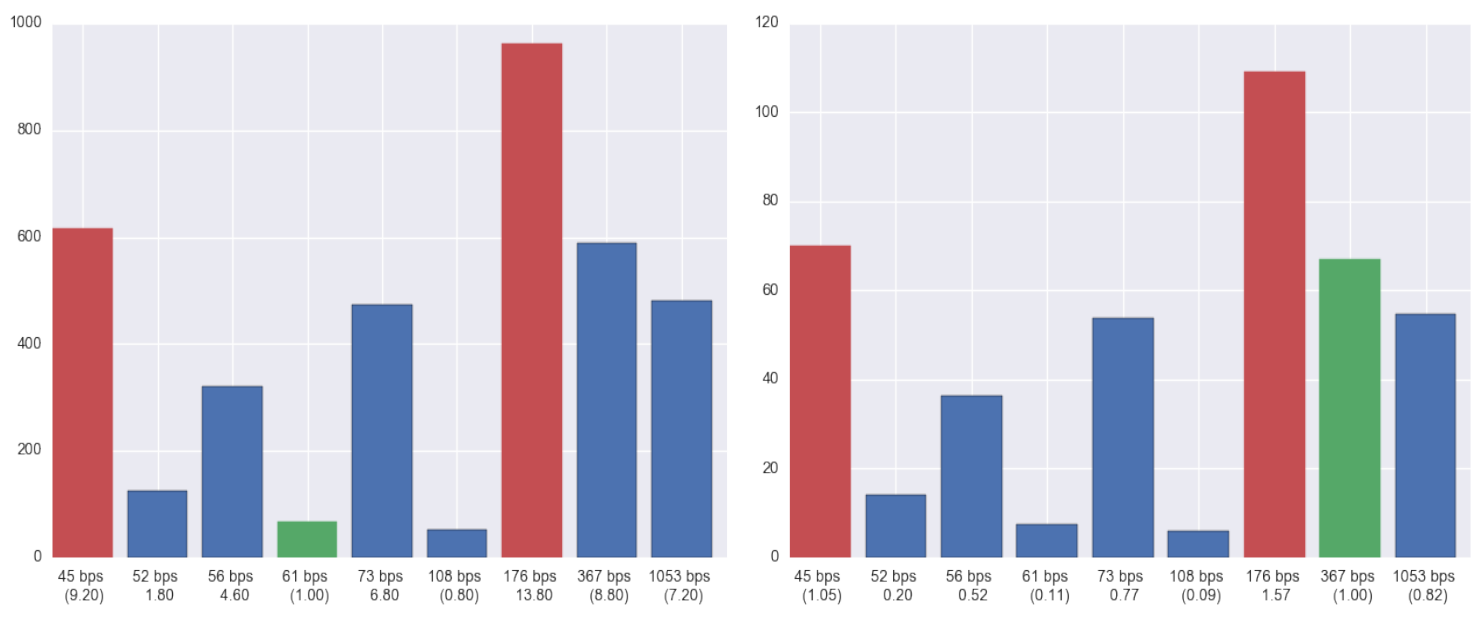

Figure 5: Time-0 EADs in basis points (IM quantile level $a=70 \%$, liquidation period $\delta=5$ days). The two largest EADs, in red, size the default fund. The reference member EAD is in green. The corresponding positions $\omega_{i}$ of the member are displayed at the bottom. Left: Reference member with $\Sigma_{0}=61$ bps and $\nu_{0}=53.00$. Right: Reference member with $\Sigma_{0}=367$ bps and $\nu_{0}=5.14$.

\subsection{XVA Data}

The following numerical values are used in the sequel:

$$
\bar{R}=1, \quad \bar{\lambda}=\frac{1}{2} \Sigma_{0}, \quad \lambda=0, \quad k=10 \%, \quad h=1 \text { day, } \quad \mu=\frac{2}{\bar{T}}, \quad m=10^{4},
$$

where $m$ is the number of simulations used for estimating the expectations in 6.4 and 6.9. The level of $10 \%$ used for $k$ is consistent with reference orders of magnitude for a hurdle rate.

Moreover, in a CCP setup, unless otherwise stated, we set

$$
\begin{aligned}
& R=0, \delta=5 \text { days }, a=70 \%, T=1 \text { month, } Y=1 \text { year, } \\
& E^{\star}=25 \% K^{c c p}, c=30 \mathrm{bp},
\end{aligned}
$$

where $K^{c c p}$ is defined in A.4 . The low quantile level used to set the initial margins is meant to compensate the excessive simplicity of the Black-Scholes setup without wrong-way risk used for $S$ (it also leads to moderate standard errors with a relatively small number $m=10^{4}$ of simulations). Margin fees of $c=30 \mathrm{bp}$ are consistent with current CCP practices. These margin fees are distinct from the commission fees, not included in our setup, that a CCP is also charging to its members. In practice, commission fees are of the order of a few basis points of the size of the positions, i.e. a few basis points in the case of a unit position in our swap with each leg equal to one at time 0.

In a CSA setup, alternatively to $(7.8)$, unless otherwise stated, we set

$$
R_{b}=R_{c}=40 \%, \delta=15 \text { days, } a=80 \%, c=0 .
$$

The value $a=80 \%$ used in the bilateral case is higher than the value $a=70 \%$ used in the CCP setup, where the protection offered by the default fund allows requiring less initial margins. 


\section{Numerical Results}

All our XVA numbers are stated in basis points (recall that both legs of the swap are worth one at time 0). For comparability purposes, common random inputs are used in all our Monte Carlo estimates, i.e. we use the same sampled trajectories of $S$ and sampled sets of default times $\tau_{i}$ in all cases, it is only the way these $m=10^{4}$ random input sets are used which changes. The computation times are proportional to the number of members $n$ and model trajectories $m$, e.g. about 5 minutes on a standard laptop to compute a full set of XVAs in Table 4 (four or five XVA components and their sum), where $n=8$ and $m=10^{4}$, using pre-simulated values for all the random inputs. Negative (e.g. DVA) numbers are displayed in parentheses. Regarding the aggregated XVA numbers in the tables, i.e. BVA in the CSA setup, CCVA in the CCP setup and TVA sometimes used as a common acronym for covering both cases, they are all KVA-inclusive, but they do not include the corresponding DVA numbers, which are only showed for reference. In other words, all the displayed TVA numbers correspond to entry price TVAs. The CCP MLA number are consistently found one order of magnitude smaller than the other XVA numbers, which is a sanity check that the CCP margin fees do not drive the comparison between the CCP and the CSA setup.

Note that, for simplicity, we are comparing a situation where all the trading is centrally cleared with a situation where all the trading is bilateral. In practice, vanilla products (hedges) tend to be cleared and exotics tend to be bilaterally traded. Therefore, in a more realistic setup, the multilateral netting benefit that CCPs provide is balanced by a loss of bilateral netting across asset classes (see Duffie and Zhu (2011) and Cont, Santos, and Moussa (2013)). To correct this bias, we will also show bilateral XVA figures scaled by the compression factor $\nu_{0}$ of the reference name.

\subsection{Multilateral Netting Benefit}

Table 4 shows the XVA numbers obtained by considering alternately each of the nine members in Table 1 as reference member, using the $\alpha_{i}$ coefficients for setting the positions of the members in each case as explained in Sect. 7.3 (cf. the examples 7.1 and 7.2). The different cases in Table 4 are ordered by increasing values of the compression factor $\nu_{0}$, i.e. by decreasing $\left|\alpha_{0}\right|$. We can see from Table 4 that the MVA and the KVA are the main contributors in the respective CSA and CCP setup. Moreover, the CSA XVA numbers vary roughly proportionally to the compression factor $\nu_{0}$, whereas the CCP XVA numbers are essentially not impacted by $\nu_{0}$. This illustrates the multilateral netting benefit provided by the CCP, especially for members with a large compression factor $\nu_{0}$. 


\begin{tabular}{|c|c|c|c|c|c|c|c|c|c|}
\hline$\nu_{0}$ & 2.91 & 4.87 & 5.14 & 6.50 & 6.94 & 10.74 & 29 & 53 & 66.50 \\
$\alpha_{0}$ & 0.69 & $(0.46)$ & $(0.44)$ & $(0.36)$ & 0.34 & 0.23 & 0.09 & $(0.05)$ & $(0.04)$ \\
$\Sigma_{0}$ & 176 & 45 & 367 & 1053 & 73 & 56 & 52 & 61 & 108 \\
\hline CVA & 11.07 & 25.06 & 19.34 & 14.06 & 28.37 & 42.69 & 111.38 & 238.22 & 299.37 \\
DVA & $(8.76)$ & $(4.49)$ & $(30.85)$ & $(90.10)$ & $(8.08)$ & $(13.59)$ & $(28.77)$ & $(52.70)$ & $(111.33)$ \\
MVA & 30.38 & 13.63 & 110.50 & 339.69 & 31.41 & 39.34 & 98.46 & 204.72 & 449.68 \\
KVA & 11.17 & 21.16 & 19.40 & 21.14 & 29.26 & 46.28 & 122.20 & 221.63 & 275.87 \\
BVA & 52.62 & 59.85 & 149.24 & 374.90 & 89.04 & 128.31 & 332.05 & 664.57 & 1024.92 \\
\hline CVA & 7.88 & 11.33 & 6.54 & 3.57 & 10.85 & 11.73 & 11.91 & 11.60 & 9.23 \\
DVA & $(2.57)$ & $(0.69)$ & $(5.43)$ & $(13.03)$ & $(1.07)$ & $(0.89)$ & $(0.81)$ & $(0.90)$ & $(1.57)$ \\
MVA & 5.19 & 1.39 & 10.33 & 24.24 & 2.22 & 1.76 & 1.61 & 1.86 & 3.23 \\
MLA & 1.17 & 1.22 & 1.09 & 0.89 & 1.22 & 1.22 & 1.22 & 1.22 & 1.20 \\
KVA & 10.79 & 11.59 & 10.00 & 7.97 & 11.44 & 11.52 & 11.54 & 11.58 & 11.21 \\
CCVA & 25.03 & 25.54 & 27.95 & 36.67 & 25.73 & 26.23 & 26.27 & 26.26 & 24.87 \\
\hline
\end{tabular}

Table 4: XVA numbers obtained by considering alternately each of the nine members in Table 1 as reference member 0 , using the $\alpha_{i}$ for setting the positions of the members in each case as explained in Sect. 7.3. ( $U p)$ Credit spread $\Sigma_{0}$, coefficient $\alpha_{0}$ and compression factor $\nu_{0}$ of the reference member in each case (ordered by increasing $\nu_{0}$, i.e. decreasing $\left.\left|\alpha_{0}\right|\right)$. (Middle) CSA XVA numbers. (Bottom) CCP XVA numbers.

Table 5 shows the percentage standard errors corresponding to the Monte Carlo estimates of Table 4. As we can see from the table, the standard errors are typically no more than a few percents in relative terms. Standard errors of Monte Carlo estimates are no longer shown in the sequel.

\begin{tabular}{|c|c|c|c|c|c|c|c|c|c|}
\hline$\nu_{0}$ & 2.91 & 4.87 & 5.14 & 6.50 & 6.94 & 10.74 & 29 & 53 & 66.50 \\
$\alpha_{0}$ & 0.69 & $(0.46)$ & $(0.44)$ & $(0.36)$ & 0.34 & 0.23 & 0.09 & $(0.05)$ & $(0.04)$ \\
$\Sigma_{0}$ & 176 & 45 & 367 & 1053 & 73 & 56 & 52 & 61 & 108 \\
\hline CVA & 3.40 & 2.87 & 3.40 & 4.97 & 3.22 & 3.22 & 3.22 & 2.90 & 2.89 \\
DVA & 5.66 & 10.38 & 4.08 & 2.58 & 8.92 & 9.21 & 9.49 & 9.28 & 7.05 \\
MVA & 0.79 & 0.78 & 0.75 & 0.96 & 0.77 & 0.64 & 0.63 & 0.84 & 0.80 \\
KVA & 0.58 & 0.54 & 0.64 & 0.81 & 0.54 & 0.54 & 0.54 & 0.54 & 0.55 \\
\hline CVA & 2.55 & 2.93 & 3.13 & 4.49 & 2.69 & 2.71 & 2.70 & 2.91 & 2.66 \\
DVA & 3.11 & 3.02 & 3.05 & 3.42 & 3.15 & 2.92 & 2.94 & 3.27 & 3.21 \\
MVA & 0.86 & 0.78 & 0.77 & 0.96 & 0.91 & 0.67 & 0.69 & 0.95 & 0.93 \\
MLA & 0.65 & 0.60 & 0.71 & 0.88 & 0.61 & 0.61 & 0.60 & 0.60 & 0.62 \\
KVA & 0.58 & 0.58 & 0.65 & 0.84 & 0.57 & 0.59 & 0.59 & 0.59 & 0.58 \\
\hline
\end{tabular}

Table 5: Percentage standard errors corresponding to the Monte Carlo estimates of Table 4.

\subsection{Impact of the Credit Spread of the Reference Member}

The CCP multilateral netting benefit dominates the comparison between our CSA and CCP XVA numbers. However, in our stylized setup, we cannot see the netting benefit across assets of bilateral trading. In order to compensate for this bias and obtain comparative results net of the first order CCP multilateral netting benefit, Table 6 shows the same results as Table 4, but with all the CSA XVA numbers scaled by the corresponding compression factor $\nu_{0}$ (we will present in this way all the CSA XVA results in the sequel) and ordered 
by increasing credit spread $\Sigma_{0}$ of the reference name, instead of increasing $\nu_{0}$ in Table 4 .

From Table 6 we can see that, if we get rid of the CCP multilateral netting benefit through this scaling, then the CSA and CCP XVA numbers become of a similar order of magnitude. The aggregated TVA numbers even become in favor of the CSA setup, except for the reference name with the largest (actually huge) credit spread of $1053 \mathrm{bp}$. These results can be put in perspective with the ones in Ghamami and Glasserman (2016) (see Sect. 1.1.

Regarding the comparison between the nine different cases within the CCP setup, as also within the CSA setup after scaling by the compression factor, Table 6 shows that the main explanatory factor of the results is the credit spread of the reference member, risky members being heavily penalized in terms of MVA, especially in the CSA setup. In both cases, the dominant patterns are a logarithmic decrease of the CVA numbers and a linear increase of the $|\mathrm{DVA}|$ and MVA numbers with respect to the credit spread of the reference name.

\begin{tabular}{|c|c|c|c|c|c|c|c|c|c|}
\hline$\nu_{0}$ & 4.87 & 29 & 10.74 & 53 & 6.94 & 66.5 & 2.91 & 5.14 & 6.5 \\
$\alpha_{0}$ & $(0.46)$ & 0.09 & 0.23 & $(0.05)$ & 0.34 & $(0.04)$ & 0.69 & $(0.44)$ & $(0.36)$ \\
$\Sigma_{0}$ & 45 & 52 & 56 & 61 & 73 & 108 & 176 & 367 & 1053 \\
\hline CVA $/ \nu_{0}$ & 5.15 & 3.84 & 3.97 & 4.49 & 4.09 & 4.50 & 3.80 & 3.76 & 2.16 \\
DVA $/ \nu_{0}$ & $(0.92)$ & $(0.99)$ & $(1.27)$ & $(0.99)$ & $(1.16)$ & $(1.67)$ & $(3.01)$ & $(6.00)$ & $(13.86)$ \\
MVA $/ \nu_{0}$ & 2.80 & 3.40 & 3.66 & 3.86 & 4.53 & 6.76 & 10.44 & 21.50 & 52.26 \\
KVA $/ \nu_{0}$ & 4.34 & 4.21 & 4.31 & 4.18 & 4.22 & 4.15 & 3.84 & 3.77 & 3.25 \\
BVA $/ \nu_{0}$ & 12.29 & 11.45 & 11.95 & 12.54 & 12.83 & 15.41 & 18.08 & 29.03 & 57.68 \\
\hline CVA & 11.33 & 11.91 & 11.73 & 11.60 & 10.85 & 9.23 & 7.88 & 6.54 & 3.57 \\
DVA & $(0.69)$ & $(0.81)$ & $(0.89)$ & $(0.90)$ & $(1.07)$ & $(1.57)$ & $(2.57)$ & $(5.43)$ & $(13.03)$ \\
MVA & 1.39 & 1.61 & 1.76 & 1.86 & 2.22 & 3.23 & 5.19 & 10.33 & 24.24 \\
MLA & 1.22 & 1.22 & 1.22 & 1.22 & 1.22 & 1.20 & 1.17 & 1.09 & 0.89 \\
KVA & 11.59 & 11.54 & 11.52 & 11.58 & 11.44 & 11.21 & 10.79 & 10.00 & 7.97 \\
CCVA & 25.54 & 26.27 & 26.23 & 26.26 & 25.73 & 24.87 & 25.03 & 27.95 & 36.67 \\
\hline
\end{tabular}

Table 6: XVA numbers obtained by considering alternately each of the nine members in Table 1 as reference member 0 , using the $\alpha_{i}$ for setting the positions of the members in each case as explained in Sect. 7.3. (Up) Credit spread $\Sigma_{0}$, coefficient $\alpha_{0}$ and compression factor $\nu_{0}$ of the reference member in each case (ordered by increasing $\Sigma_{0}$ ). (Middle) CSA XVA numbers scaled by the compression factors $\nu_{0}$. (Bottom) CCP XVA numbers.

\subsection{Impact of the Liquidation Period}

Focusing on the reference members of the examples 7.1 and 7.2 , respectively dubbed "safe member" and "risky member" henceforth (with respective credit spread of $\Sigma_{0}=61$ and $367 \mathrm{bp}$ ), Table 7 shows the impact of changing the length $\delta$ of the liquidation period from 5 days to 15 days in the CSA setup and vice versa in the CCP setup. The CSA 15 day and CCP 5 day numbers in Table 7 are simply retrieved from Table 6 , for comparison purposes with the additional CSA 5 day and CCP 15 day numbers. The results are consistent with a $\sqrt{\delta}$ pattern in line with the distributional properties of the Black-Scholes model used for $S$. 


\begin{tabular}{|c|c|c|c|c|}
\hline Member & \multicolumn{2}{|c|}{$61 \mathrm{bps}, \nu_{0}=53.00$} & \multicolumn{2}{c|}{$367 \mathrm{bps}, \nu_{0}=5.14$} \\
\hline$\delta$ & $5 \mathrm{~d}$ & $15 \mathrm{~d}$ & $5 \mathrm{~d}$ & $15 \mathrm{~d}$ \\
\hline $\mathrm{CVA} / \nu_{0}$ & 2.17 & 4.49 & 1.82 & 3.76 \\
$\mathrm{DVA} / \nu_{0}$ & $(0.50)$ & $(0.99)$ & $(2.90)$ & $(6.00)$ \\
$\mathrm{MVA} / \nu_{0}$ & 2.34 & 3.86 & 13.14 & 21.50 \\
$\mathrm{KVA} / \nu_{0}$ & 2.43 & 4.18 & 2.18 & 3.77 \\
$\mathrm{BVA} / \nu_{0}$ & 6.94 & 12.54 & 17.15 & 29.03 \\
\hline $\mathrm{CVA}$ & 11.60 & 19.54 & 6.54 & 10.78 \\
$\mathrm{DVA}$ & $(0.90)$ & $(1.41)$ & $(5.43)$ & $(8.91)$ \\
$\mathrm{MVA}$ & 1.86 & 3.40 & 10.33 & 18.96 \\
$\mathrm{MLA}$ & 1.22 & 2.25 & 1.09 & 2.00 \\
KVA & 11.58 & 21.60 & 10.00 & 18.59 \\
CCVA & 26.26 & 46.79 & 27.95 & 50.34 \\
\hline
\end{tabular}

Table 7: Impact of the liquidation period. (Left) Safe reference member. (Right) Risky reference member. (Top) CSA XVA numbers scaled by $\nu_{0}$. (Bottom) CCP XVA numbers.

\subsection{Margin Optimization}

Table 8 shows the impact of using higher quantile levels $a$ for the initial margins, which were only $80 \%$ and $70 \%$ in the respective CSA and CCP setup so far (with the motivation exposed in Sect. 7.6). The left column in each of the two main panels, retrieved from Table 6. corresponds to our base case where $a=70 \%$ and $a=80 \%$. When higher values are used for the quantile levels, i.e. going from left to right in each panel, we observe the same qualitative patterns as before in terms of the comparison between the CSA and the CCP setup. Considering now the impact of higher quantile levels inside each CSA or CCP setup, we can see a shift from CVA(/DVA) and KVA into MVA.

Ultimately, for very high quantiles, the CVA(/DVA) and KVA would reach zero whereas the MVA would keep increasing, since excessive margins become useless and a pure cost to the system, in the CSA as in the CCP setup. Figure6illustrates this further by showing the aggregated TVA numbers and the relative weight of their CVA, FVA and KVA contributions when the quantile level $a$ used for setting the IM goes from $55 \%$ to $100 \%$, where FVA means MVA in the CSA setup (left graphs) and MVA+MLA in the CCP setup (right graphs). In each of the four cases considered in the upper panels (left CSA vs. right CCP curve and blue safe vs. green risky reference member curve), the numerical values of the TVA exhibit a convex dependence with respect to $a$ (although, mathematically speaking, this depends on the values of the numerical parameters that are used, see for instance the CVA curve in the left graph of Figure 7, which shows a more detailed XVA decomposition of the safe reference member CCVA curve in the upper right graph of Figure 6). In the case of the risky reference member in the CSA setup, the level of initial margins is too high already with a $55 \%$ quantile level: The risky reference member (green) BVA curve in the upper left graph of Figure 6 keeps increasing when $a$ increases from $55 \%$ to $100 \%$. In each of the other three cases, the TVA has a minimum at some value $a<1$. For both reference names, the optimal quantile level is larger in the CCP than in the CSA setup. This is because, in the CCP setup, the member is happy to post more initial margins, which "cost" her $\bar{\lambda}=\frac{1}{2} \Sigma_{0}$, in order to reduce her default fund contribution, which "costs" her a greater $k=10 \%$ (cf. (7.7)). In each of the four considered cases, the FVA becomes preponderant 
and even hegemonic (as it tends to infinity) when $a$ goes to $100 \%$.

Capponi and Cheng (2016) construct a model which endogenizes collateral, making it part of an optimization problem where the CCP maximizes profit by controlling collateral and fee levels. They conclude that the collateral level should decrease with funding costs, on top of increasing with market volatility. The above numerical results are quite in line with such statements.

\begin{tabular}{|c||c|c|c||c|c|c|}
\hline \multicolumn{1}{|c||}{ Member } & \multicolumn{3}{c||}{$\Sigma_{0}=61 \mathrm{bp}, \nu_{0}=53.00$} & \multicolumn{3}{c|}{$\Sigma_{0}=367 \mathrm{bp}, \nu_{0}=5.14$} \\
\hline $\mathrm{a}$ & $80 \%$ & $90 \%$ & $99 \%$ & $80 \%$ & $90 \%$ & $99 \%$ \\
\hline CVA $/ \nu_{0}$ & 4.49 & 2.64 & 0.74 & 3.76 & 2.23 & 0.62 \\
DVA $/ \nu_{0}$ & $(0.99)$ & $(0.56)$ & $(0.15)$ & $(6.00)$ & $(3.51)$ & $(1.02)$ \\
MVA $/ \nu_{0}$ & 3.86 & 5.87 & 10.66 & 21.50 & 32.99 & 60.18 \\
KVA $/ \nu_{0}$ & 4.18 & 1.78 & 0.13 & 3.77 & 1.61 & 0.12 \\
BVA $/ \nu_{0}$ & 12.54 & 10.29 & 11.53 & 29.03 & 36.83 & 60.92 \\
\hline a & $70 \%$ & $80 \%$ & $95 \%$ & $70 \%$ & $80 \%$ & $95 \%$ \\
\hline CVA & 11.60 & 9.15 & 4.64 & 6.54 & 5.17 & 2.62 \\
DVA & $(0.90)$ & $(0.66)$ & $(0.22)$ & $(5.43)$ & $(4.02)$ & $(1.43)$ \\
MVA & 1.86 & 2.83 & 5.32 & 10.33 & 15.71 & 29.53 \\
MLA & 1.22 & 1.54 & 2.56 & 1.09 & 1.38 & 2.31 \\
KVA & 11.58 & 6.55 & 1.19 & 10.00 & 5.66 & 1.03 \\
CCVA & 26.26 & 20.07 & 13.72 & 27.95 & 27.91 & 35.49 \\
\hline
\end{tabular}

Table 8: Impact of the level of the quantile level $a$ that is used for setting the initial margins. (Left) Safe reference member. (Right) Risky reference member. (Top) CSA setup with all XVA numbers scaled by $\nu_{0}$. (Bottom) CCP setup.

\subsection{Impact of the Number of Members}

Another interesting question is what happens when we vary the number of members of the CCP. Obviously, more members means more mutualization of risk. However, the main effects in a CCP are already visible with nine members as above: with more members, things would mainly happen as in the projection of the system onto the ten (or so) greatest members anyway. Figure 7 illustrates that, if there are now not enough members, a regulatory "cover two" default fund specification sized to the two largest exposures of the clearing members may result in very heavy default fund contributions and KVA for the small members in the common situation of heterogeneous members' exposure.

\section{Conclusions}

\section{A Regulatory Capital and Default Fund Formulas}

A primitive of all the regulatory capital formulas are the so-called exposure-at-defaults given, for $i \in N=0,1 \ldots, n$ and $t \in[0, \bar{T}]$, as

$$
E A D_{t}^{i}=\quad 1.4 \epsilon \sum_{\epsilon p<1 \wedge(T-t)} E E E_{t}^{i}\left(t_{p}\right),
$$

where (see Bank for International Settlements (2005, formulas (1)-(2)-(3) pages 26-27)): 

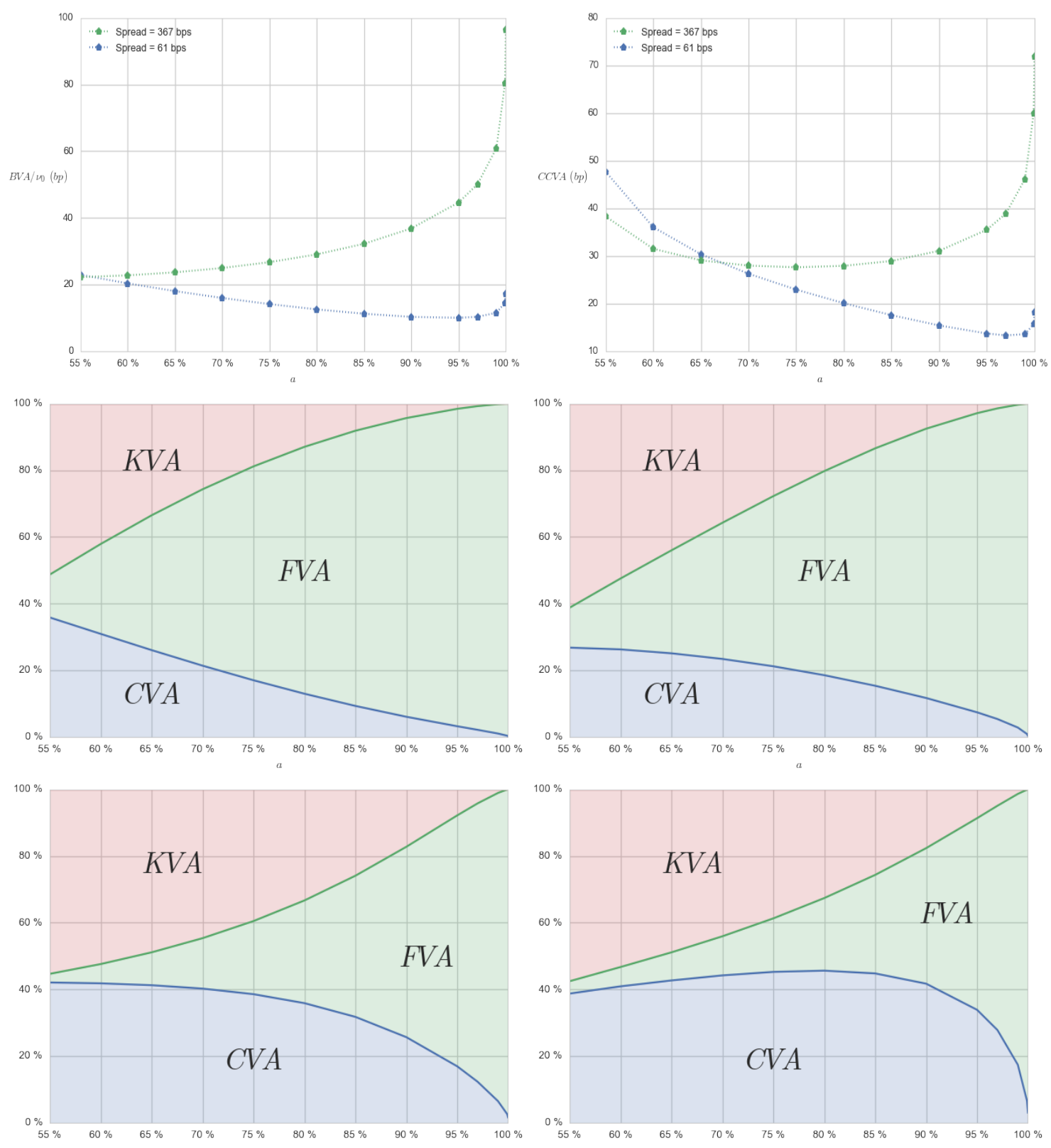

Figure 6: Varying the initial margins quantile level a. Left: CSA setup. Right: CCP setup. Top: BVA $/ \nu_{0}$ vs. CCVA. Bottom: XVA relative contributions in the case of the safe reference member. Middle: XVA relative contributions in the case of the risky reference member. 

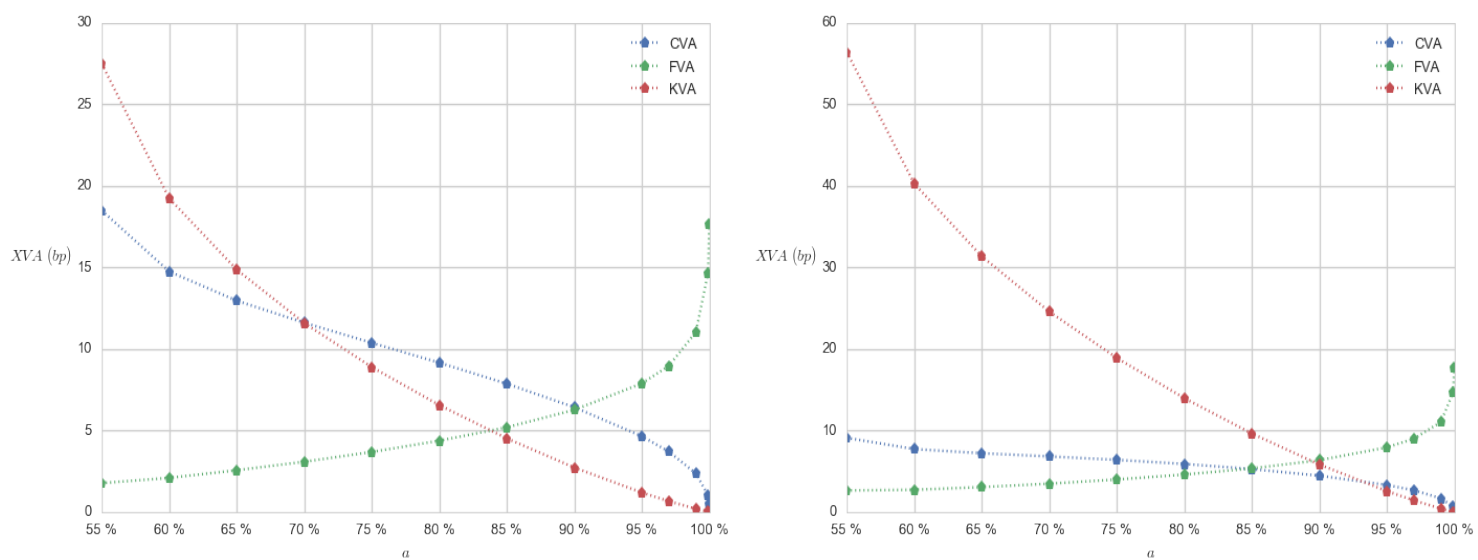

Figure 7: CCP XVA results for the reference member with $\Sigma_{0}=61 \mathrm{bp}$ and $\nu_{0}=53.00$. Left: Results in our previous CCP with nine members. Right: Results in a CCP restricted to three members: the reference member and two other members. The reference member, with $\omega_{i}=-1$ by definition, corresponds to the member with time- 0 EAD displayed in green in the left panel of Figure 5. The two other members are the members of the original CCP with the greatest time-0 EADs, i.e. the members with the time-0 EADs displayed in red in the left panel of Figure 5. Moreover, we modified the positions of these two members as $\omega_{i}=-9$ and 10, instead of -9.2 and 13.8 in the left panel of Figure 5 , for being in line with the clearing condition $\sum_{i \in N} P^{i}=0$.

- the factor 1.4 is a wrong-way risk multiplier,

- $\epsilon$ is a time-integration step (e.g. one month),

- $t_{p}=t+\epsilon p$,

- the effective expected exposures $E E E_{t}^{i}\left(t_{p}\right)$ are defined through the following iteration: $E E E_{t}^{i}\left(t_{-1}\right)=0$ and, for $p \geq 0$,

$$
E E E_{t}^{i}\left(t_{p}\right)=\max \left(E E E_{t}^{i}\left(t_{p-1}\right), \mathbb{E}_{t}\left[\left(L_{t_{p}, t_{p}+\delta^{\prime}}^{i}-I M_{t_{p}}^{i}\right)^{+}\right]\right)
$$

where $L_{t_{p}, t_{p}+\delta^{\prime}}^{i}$ has been defined in 3.2 .

In our case, we also use EADs as a proxy of the exposure of the CCP to the members in the context of EMIR "cover two" default fund computations (see Sect. A.1). For our default fund and KVA computations, such EADs must then be computed at any randomization time $t=\zeta$ used in (6.4) or for simulating the time integral in (4.14). Unless an explicit formula is available for the conditional expectations in the right-hand side of (A.2), such EAD exposures can only be done by means of nested Monte Carlo simulations.

Note that in both our centrally cleared and bilateral trading setups, we neglect capital for market risk in the paper, as if the reference member (or bank) was perfectly hedged in terms of market risk. Otherwise one more capital term is required for market risk. 


\section{A.1 CCP Setup}

Under centrally cleared trading, the "cover two" EMIR rule prescribes to size the default fund as, at least, the maximum of the greatest and of the sum of the second and third greatest exposures "under extreme but plausible market conditions" (see European Parliament (2012, article 42, paragraph 3, page 37)). This total amount is then allocated between the clearing members according to some repartition key, e.g. proportional to their initial margins.

As explained in the paper, default fund contributions are "implicit capital" that the clearing members put at the disposal of the CCP. In addition, to cover their residual risk beyond the guarantee provided by the different margin layers of the CCP, the regulatory capital $K=K^{c m}$ of a generic reference member is defined, following Basel Committee on Banking Supervision (2014, page 11), as:

$$
K^{c m}=\max \left(K^{c c p} \times \frac{D F C}{E+\sum_{i \in N} J^{i} D F C^{i}}, 8 \% \times 2 \% \times D F C\right),
$$

where DFC is the default fund contribution of the reference member and where

$$
K^{c c p}=R W \times C a p_{R a t i o} \times \sum_{i \in N} J^{i} E A D^{i}
$$

with $R W=20 \%$ and Cap $_{\text {Ratio }}=8 \%$.

Remark A.1 Ghamami (2015) argues that the CCP regulatory capital $K^{\mathrm{cm}}$ of a member should rather be based on its expected future unfunded default fund contributions (see the remark 3.4), which represent the losses of the member beyond the level already funded via its default fund contribution.

\section{A.2 CSA Setup}

In the bilateral setup, the capital at risk $K$ of the bank reduces to its regulatory capital (there is no bilateral trading analog of the default fund), which comprises a first contribution for counterparty default losses and a second one for the volatility of the CVA (the market risk of the bank being supposed to be hedged out). Since we focus on the reference member 0 with $n$ counterparties $i \in N^{\star}=\{1,2, \ldots, n\}$, the capital formulas below all need to be summed over $i \in N^{\star}$.

\section{A.2.1 $K^{c c r}$}

The Basel II regulatory capital specified for counterparty risk is defined as

$$
K^{c c r}=\operatorname{Cap}_{\text {Ratio }} \sum_{i \in N^{\star}} R W A^{i},
$$

where

$$
R W A^{i}=12.5 \times w_{i} \times 1.4 \times E A D^{i} .
$$

Here Cap $_{\text {Ratio }} \geq 8 \%$ (which is the value that we use in the numerics) is a chosen capital ratio that the bank must hold. The capital weight $w_{i}$ is given by the internal ratings-based formula

$$
w_{i}=\left(1-R_{i}\right)\left(\Phi\left(\frac{\Phi^{-1}\left(D P_{i}\right)}{\sqrt{1-\operatorname{corr}_{i}}}+\sqrt{\frac{\operatorname{corr}_{i}}{1-\operatorname{corr}_{i}}} \Phi^{-1}(0.999)\right)-D P_{i}\right) \frac{1+\left(\widehat{T}^{i}-2.5\right) b\left(D P_{i}\right)}{1-1.5 b\left(D P_{i}\right)}
$$


(see Basel Committee on Banking Supervision (2005, page 7)), where:

- $R_{i}$ is the recovery rate of the counterparty $i$,

- $\Phi$ is the standard normal cdf,

- $D P_{i}$ is the one year default probability of the counterparty $i$, historical in principle, proxied in our numerics by the risk-neutral default probability extracted from the corresponding CDS spread,

- corr $_{i}$ is the asset-counterparty $i$ correlation in the sense of

$$
\operatorname{corr}_{i}=0.12 \frac{1-e^{-50 D P_{i}}}{1-e^{-50}}+0.24 \frac{1-\left(1-e^{-50 D P_{i}}\right)}{1-e^{-50}}
$$

- $\widehat{T}^{i}$ is the effective time to maturity of the netting set $i$, i.e. the time to maturity of the swap in our numerical case study where a single derivative is considered,

- $b(p)=(0.11852-0.05478 \ln (p))^{2}$.

\section{A.2.2 $K^{c v a}$}

The standardized CVA risk capital charge in Basel Committee on Banking Supervision $(2011, \S 104)$ reads as

$$
K^{c v a}=2.33 \sqrt{Y}\left[\left(0.5 \sum_{i \in N^{\star}} w_{i} \widehat{T}^{i} \widetilde{E A D}^{i}\right)^{2}+0.75 \sum_{i \in N^{\star}}\left(w_{i} \widehat{T}^{i} \widetilde{E A D}^{i}\right)^{2}\right]^{0.5},
$$

which we approximate as in Green, Kenyon, and Dennis (2014) by

$$
\frac{2.33}{2} \sqrt{Y} \sum_{i \in N^{\star}} w_{i} \widehat{T}^{i} \widetilde{E A D}^{i}
$$

where:

- $Y$ is the one year risk horizon, i.e. $Y=1$,

- $\widehat{T}^{i}$ is above,

- $\widetilde{E A D}^{i}=\frac{1-\exp \left(0.05 \widehat{T}^{i}\right)}{0.05 \widehat{T}^{i}} E A D^{i}$,

- $w_{i}$ is a weight based on the external rating extracted from the one year default probability $D P_{i}$ as of the following table, where the left part comes from Moody's and the right part is taken from Basel Committee on Banking Supervision (2011, §104);

\begin{tabular}{|c|c|c|}
\hline Default Prob & Rating & Weight \\
\hline $0.00 \%$ & AAA & $0.7 \%$ \\
$0.02 \%$ & AA & $0.7 \%$ \\
$0.06 \%$ & A & $0.8 \%$ \\
$0.17 \%$ & BBB & $1.0 \%$ \\
$1.06 \%$ & BB & $2.0 \%$ \\
$3.71 \%$ & B & $3.0 \%$ \\
$12.81 \%$ & CCC & $10.0 \%$ \\
\hline
\end{tabular}




\section{B Proofs of Auxiliary Results}

\section{B.1 Proof of Lemma 3.1}

Under our stylized model of the liquidation procedure, during the liquidation period $\left[\tau_{Z}, \tau_{Z}^{\delta}\right]$, where $\tau_{Z}=\tau_{i}$ if and only if $i \in Z$, the clearing house substitutes itself to the defaulting members, taking care of all their dividend cash flows, which represent a cumulative cost of $\sum_{i \in Z} \Delta_{\tau_{i}^{\delta}}^{i}$ (including a funding cost at the risk-free rate comprised in the $\Delta_{\tau_{i}^{\delta}}^{i}$ ). At the liquidation time $\tau_{Z}^{\delta}$, the clearing house substitutes the buffer to itself as counterparties in all the concerned contracts (or simply puts an end to the contracts that were already with the buffer), which represents a supplementary cost $\sum_{i \in Z} P_{\tau_{i}^{\delta}}^{i}$. In addition, for any $i \in Z$ :

- If $\varepsilon_{i}=0$, meaning that $Q_{\tau_{i}^{\delta}}^{i} \leq \mathcal{C}_{\widehat{\tau}_{i}}^{i}$, then either $Q_{\tau_{i}^{\delta}}^{i} \leq 0$ and an amount $\left(-Q_{\tau_{i}^{\delta}}^{i}\right)$ is paid by the clearing house to the member $i$ (who keeps ownership of all its collateral), or $Q_{\tau_{i}^{\delta}}^{i} \geq 0$ and the ownership of an amount $Q_{\tau_{i}^{\delta}}^{i}$ of collateral is transferred to the clearing house. In both cases, the clearing house gets $Q_{\tau_{i}^{\delta}}^{i}$;

- Else, i.e. if $\varepsilon_{i}>0$, meaning that the overall collateral $\mathcal{C}^{i}$ of a member $i \in Z$ does not cover the totality of its debt to the clearing house, then, at time $\tau_{i}^{\delta}$, the ownership of $\mathcal{C}^{i}$ is transferred in totality to the clearing house. If $R_{i}>0$ then the clearing house also gets a recovery $R_{i} \varepsilon_{i}$.

In conclusion, the realized breach of the $\mathrm{CCP}$ is the sum over $i \in Z$ of the

$$
\begin{gathered}
P_{\tau_{i}^{\delta}}^{i}+\Delta_{\tau_{i}^{\delta}}^{i}-\mathbb{1}_{\varepsilon_{i}>0}\left(\mathcal{C}_{\widehat{\tau}_{i}}^{i}+R_{i} \varepsilon_{i}\right)-\mathbb{1}_{\varepsilon_{i}=0} Q_{\tau_{i}^{\delta}}^{i}=Q_{\tau_{i}^{\delta}}^{i}-\mathbb{1}_{\varepsilon_{i}=0} Q_{\tau_{i}^{\delta}}^{i}-\mathbb{1}_{\varepsilon_{i}>0}\left(\mathcal{C}_{\widehat{\tau}_{i}}^{i}+R_{i} \varepsilon_{i}\right) \\
=\mathbb{1}_{\varepsilon_{i}>0}\left(Q_{\tau_{i}^{\delta}}^{i}-\mathcal{C}_{\widehat{\tau}_{i}}^{i}-R_{i} \varepsilon_{i}\right)=\left(1-R_{i}\right) \varepsilon_{i}=\xi_{i} .
\end{gathered}
$$

\section{B.2 Proof of Lemma 4.1}

To formulate in mathematical terms the above-described margining, hedging and funding policy of the member, we introduce three funding assets $B^{0}, B^{f}$ and $\bar{B}^{f}$ evolving on $\left[0, \bar{\tau}^{\delta}\right]$ as

$$
d B_{t}^{0}=r_{t} B_{t}^{0} d t, d B_{t}^{f}=\left(r_{t}+\lambda_{t}\right) B_{t}^{f} d t, d \bar{B}_{t}^{f}=\left(r_{t}+\bar{\lambda}_{t}\right) \bar{B}_{t}^{f} d t+(1-\bar{R}) \bar{B}_{t-}^{f} d J_{t} .
$$

These represent the risk-free OIS deposit asset and the assets used by the bank for its respective investing and unsecured funding purposes. Under our continuous-time mark-tomodel and realization assumption on profit-and-losses, the amount on the funding accounts of the bank is

$$
-\Pi_{t}=-\left(\Pi_{t}+\mathcal{C}_{t}^{\star}\right)+\mathcal{C}_{t}^{\star}
$$

where $\mathcal{C}_{t}^{\star}=V M+I M$ is the amount of margins that need to be funded by the member (its default fund contribution is assumed to be taken on its uninvested equity, hence does not need to be funded), so that the terms in the parenthesis represent the amount invested or 
borrowed unsecured (depending on its sign) by the bank, and where we recall that collateral is remunerated OIS by the receiving party. Defining

$$
\eta_{t}^{f}=\frac{\left(\Pi_{t}+\mathcal{C}_{t}^{\star}\right)^{-}}{B_{t}^{f}}, \quad \bar{\eta}_{t}^{f}=-\frac{\left(\Pi_{t}+\mathcal{C}_{t}^{\star}\right)^{+}}{\bar{B}_{t}^{f}}, \quad \eta_{t}^{0}=\frac{\mathcal{C}_{t}^{\star}}{B_{t}^{0}}, \quad \bar{\eta}_{t}^{0}=-\frac{\left(\Pi_{t}+\mathcal{C}_{t}^{\star}\right)}{B_{t}^{0}},
$$

we can write

$$
-\Pi_{t}=J_{t} \eta_{t}^{f} B_{t}^{f}+J_{t} \bar{\eta}_{t}^{f} \bar{B}_{t}^{f}+\eta_{t}^{0} B_{t}^{0}+\left(1-J_{t}\right) \bar{\eta}_{t}^{0} B_{t}^{0},
$$

where, by self-financing condition,

$$
\begin{aligned}
d\left(J_{t} \eta_{t}^{f} B_{t}^{f}+J_{t} \bar{\eta}_{t}^{f} \bar{B}_{t}^{f}+\eta_{t}^{0} B_{t}^{0}+\left(1-J_{t}\right) \bar{\eta}_{t}^{0} B_{t}^{0}\right) \\
=J_{t} \eta_{t}^{f} d B_{t}^{f}+J_{t-} \bar{\eta}_{t-}^{f} d \bar{B}_{t}^{f}+\eta_{t}^{0} d B_{t}^{0}+\left(1-J_{t}\right) \bar{\eta}_{t}^{0} d B_{t}^{0}
\end{aligned}
$$

A left-limit in time is required in $J_{t-} \bar{\eta}_{t-}^{f}$ because $\bar{B}_{t}^{f}$ in (B.1) jumps at time $\tau$, so that the process $\bar{\eta}^{f}$, which is defined through $(\overline{\mathrm{B} .2})$, is not predictable.

In view of $(\mathrm{B} .3)-(\mathrm{B} .4)$ and of the additional cash flows that affect the member (contractual cash flows, margin fees, realized breaches refills and hedging cash flows), the gain process $e$ associated with the member's valuation-and-hedge policy $(\Pi, \zeta)$ satisfies the following forward SDE: $e_{0}=0$ and, for $0<t \leq \bar{\tau}^{\delta}$,

$$
\begin{aligned}
& d e_{t}=\underbrace{d \Pi_{t}}_{\text {gain on the derivative portfolio }}-\underbrace{J_{t} d D_{t}}_{\text {contractual dividends }}-\underbrace{J_{t} c_{t}\left(\mathcal{C}_{t}-P_{\widehat{t}-}\right) d t}_{\text {margin fees }} \\
& -\underbrace{J_{t} \sum_{Z \subseteq N} \epsilon_{\tau_{Z}^{\delta}} \boldsymbol{\delta}_{\tau_{Z}^{\delta}}(d t)}_{\text {loss on the hedge }}-\underbrace{\zeta_{t} d \mathcal{M}_{t}} \\
& \text { refill of realized breaches } \\
& +J_{t} \eta_{t}^{f} d B_{t}^{f}+J_{t-} \bar{\eta}_{t-}^{f} d \bar{B}_{t}^{f}+\eta_{t}^{0} d B_{t}^{0}+\left(1-J_{t}\right) \bar{\eta}_{t}^{0} d B_{t}^{0} .
\end{aligned}
$$

Substituting (B.1) into the above yields

$$
\begin{aligned}
& d e_{t}=d \Pi_{t}-r_{t} \Pi_{t} d t-\zeta_{t} d \mathcal{M}_{t}-\mathbb{1}_{\{\tau<\bar{T}\}}(1-\bar{R})\left(\Pi+\mathcal{C}_{\hat{t}}^{\star}\right)^{+} d J_{t} \\
& -J_{t}\left(d D_{t}+\sum_{Z \subseteq N} \epsilon_{\tau_{Z}^{\delta}} \delta_{\tau_{Z}^{\delta}}(d t)+\left(c_{t}\left(\mathcal{C}_{t}-P_{\widehat{t}-}\right)+\bar{\lambda}_{t}\left(\Pi_{t}+\mathcal{C}_{t}^{\star}\right)^{+}-\lambda_{t}\left(\Pi_{t}+\mathcal{C}_{t}^{\star}\right)^{-}\right) d t\right),
\end{aligned}
$$

which is (4.3), by definition (4.4) of $g$.

\section{B.3 Proof of Lemma 5.1}

Since $\xi=(1-R)\left(Q_{\tau^{\delta}}-\mathcal{C}_{\widehat{\tau}}\right)^{+}($cf. $(3.8))$, where

$$
\begin{aligned}
& \mathcal{C}_{\widehat{\tau}}=\mathcal{C}_{\tau-}=\mathcal{C}\left(\tau, X_{\tau-}\right) \text { and } \\
& Q_{\tau^{\delta}}=P_{\tau^{\delta}}+\Delta_{\tau^{\delta}}=P\left(\tau^{\delta}, \mathbf{X}_{\tau^{\delta}}\right)+\widehat{\Delta}\left(\tau^{\delta}, \mathbf{X}_{\tau^{\delta}}\right)-e^{\int_{\tau}^{\tau^{\delta}} r\left(u, \mathbf{X}_{u}\right) d u} \widehat{\Delta}\left(\tau, \mathbf{X}_{\tau}\right),
\end{aligned}
$$

we have by definition 4.7) of $\bar{\xi}$ :

$$
\begin{aligned}
& \bar{\xi}_{\tau}=(1-R) \mathbb{E}\left[e^{-\int_{\tau}^{\tau^{\delta}} r\left(u, \mathbf{X}_{u}\right) d u} \times\right. \\
& \left.\left(P\left(\tau^{\delta}, \mathbf{X}_{\tau^{\delta}}\right)+\widehat{\Delta}\left(\tau^{\delta}, \mathbf{X}_{\tau^{\delta}}\right)-e^{\int_{\tau}^{\tau^{\delta}} r\left(u, \mathbf{X}_{u}\right) d u} \widehat{\Delta}\left(\tau, \mathbf{X}_{\tau}\right)-\mathcal{C}\left(\tau, X_{\tau-}\right)\right)^{+} \mid \mathcal{G}_{\tau}\right] .
\end{aligned}
$$


Therefore, the Markov property of $\mathbf{X}$ and the continuity of $\mathbf{X}$ at time $\tau$ imply that $\bar{\xi}_{\tau}$ can be represented in functional form as $\bar{\xi}\left(\tau, X_{\tau-}\right)$. Hence (cf. Crépey and Song (2016, Lemma 5.1)), it holds that

$$
\gamma_{t} \widehat{\xi}_{t}=\gamma_{t} \bar{\xi}\left(t, X_{t}\right), \mathbb{Q} \times \boldsymbol{\lambda}-\text { a.e. },
$$

where (5.1) yields $\gamma=J_{-} \gamma_{\bullet}$. This gives the result since $d v a=-\gamma \widehat{\xi}$.

\section{B.4 Proof of Lemma 6.1}

We denote by $\mathcal{T}_{\delta}$ the transition function of the homogeneous Markov process $\left(t, \mathbf{X}_{t}, \beta_{t}\right)$ over the time horizon $\delta$, i.e.

$$
(\varphi,(t, \mathbf{x}, b)) \rightarrow \mathcal{T}_{\delta}[\varphi](t, \mathbf{x}, b)=\mathbb{E}\left[\varphi\left(t^{\delta}, \mathbf{X}_{t^{\delta}}, \beta_{t^{\delta}}\right) \mid \mathbf{X}_{t}=\mathbf{x}, \beta_{t}=b\right]=\mathbb{E}\left[\varphi\left(t^{\delta}, \mathbf{X}_{t^{\delta}}, \beta_{t^{\delta}}\right) \mid \mathcal{G}_{t}\right]
$$

Recalling (B.5) and using the fact that $\mathbf{X}$ does not jump at time $\tau$, we have

$$
\bar{\xi}_{\tau}=\mathcal{T}_{\delta}\left[\xi_{\star}\left(\cdot, \cdot, \cdot, \beta_{\tau}, \mathcal{C}_{\tau-}^{\star}, \widehat{\Delta}_{\tau-}\right)\right]\left(\tau, \mathbf{X}_{\tau}, \beta_{\tau}\right)=\mathcal{T}_{\delta}\left[\xi_{\star}\left(\cdot, \cdot, \cdot, \beta_{\tau}, \mathcal{C}_{\tau-}^{\star}, \widehat{\Delta}_{\tau-}\right)\right]\left(\tau, \mathbf{X}_{\tau-}, \beta_{\tau}\right),
$$

where we set

$$
\xi_{\star}\left(t, \mathbf{x}, b, \beta_{\tau}, \mathcal{C}_{\tau-}^{\star}, \widehat{\Delta}_{\tau-}\right)=(1-R) \beta_{\tau}^{-1} b\left(P(t, \mathbf{x})+\widehat{\Delta}(t, \mathbf{x})-\beta_{\tau} b^{-1} \widehat{\Delta}_{\tau-}-\mathcal{C}_{\tau-}^{\star}\right)^{+},
$$

in which $\beta_{\tau}, \mathcal{C}_{\tau-}^{\star}$ and $\widehat{\Delta}_{\tau-}$ are considered as $\mathcal{G}_{\tau-}$ measurable parameters. In view of (B.6), we have (cf. Crépey and Song (2016, Lemma 5.1))

$$
-d v a_{t}=\gamma_{t} \widehat{\xi}_{t}=J_{t-} \gamma_{t} \mathcal{T}_{\delta}\left[\xi_{\star}\left(\cdot, \cdot, \cdot, \beta_{t}, \mathcal{C}_{t}^{\star}, \widehat{\Delta}_{t-}\right)\right]\left(t, \mathbf{X}_{t-}, \beta_{t}\right), \quad \mathbb{Q} \times \boldsymbol{\lambda} \text { a.e.. }
$$

As a consequence, given an independent random variable $\zeta$ with density $p$, we can write, using (B.7), the definition of $\mathcal{T}_{\delta}$ and (5.1) to pass to the second, third and fourth line, respectively:

$$
\begin{aligned}
-\mathbb{E}\left[h_{\zeta} \mathbb{1}_{\{\zeta \leq \bar{\tau}\}} \beta_{\zeta} d v a\left(\zeta, X_{\zeta}\right)\right]=-\int_{0}^{T} \mathbb{E}\left[h_{t} \beta_{t} \mathbb{1}_{\{t<\bar{\tau}\}} d v a\left(t, X_{t}\right)\right] p(t) d t \\
=\int_{0}^{T} \mathbb{E}\left[h_{t} \beta_{t} \mathbb{1}_{\{t \leq \tau\}} \gamma_{t} \mathcal{T}_{\delta}\left[\xi_{\star}\left(\cdot, \cdot, \cdot, \beta_{t}, \mathcal{C}_{t}^{\star}, \widehat{\Delta}_{t}\right)\right]\left(t, \mathbf{X}_{t}, \beta_{t}\right)\right] p(t) d t \\
=\int_{0}^{T} \mathbb{E}\left[h_{t} \beta_{t} \mathbb{1}_{\{t \leq \tau\}} \gamma_{t} \mathbb{E}\left[\xi_{\star}\left(t^{\delta}, \mathbf{X}_{t^{\delta}}, \beta_{t^{\delta}}, \beta_{t}, \mathcal{C}_{t}^{\star}, \widehat{\Delta}_{t}\right) \mid \mathcal{G}_{t}\right]\right] p(t) d t \\
=\int_{0}^{T} \mathbb{E}\left[h_{t} \beta_{t} \mathbb{1}_{\{t \leq \tau\}} \gamma_{\bullet}(t) \xi_{\star}\left(t^{\delta}, \mathbf{X}_{t^{\delta}}, \beta_{t^{\delta}}, \beta_{t}, \mathcal{C}_{t}^{\star}, \widehat{\Delta}_{t}\right)\right] p(t) d t \\
=\mathbb{E}\left[\mathbb{1}_{\{\zeta \leq T\}} h_{\zeta} \beta_{\zeta} \mathbb{1}_{\{\zeta \leq \tau\}} \gamma_{\bullet}(\zeta) \xi_{\star}\left(\zeta^{\delta}, \mathbf{X}_{\zeta^{\delta}}, \beta_{\zeta^{\delta}}, \beta_{\zeta}, \mathcal{C}_{\zeta}^{\star}, \widehat{\Delta}_{\zeta}\right)\right] .
\end{aligned}
$$

Acknowledgements This paper greatly benefited from regular exchanges with the quantitative research team of LCH in Paris, Quentin Archer and Julien Dosseur in particular.

\section{References}

Albanese, C. and L. Andersen (2015). Note on FVA accounting. Risk Magazine, September. 
Albanese, C., L. Andersen, and S. Iabichino (2015). FVA: Accounting and risk management. Risk Magazine, February 64-68.

Albanese, C., S. Caenazzo, and S. Crépey (2016). Capital valuation adjustment and funding valuation adjustment. arXiv:1603.03012 and ssrn.2745909 (short version "Capital and funding" published in Risk Magazine May 2016, 71-76).

Amini, H., D. Filipović, and A. Minca (2015). Systemic risk and central clearing counterparty design. ssrn2275376.

Andersen, L., D. Duffie, and Y. Song (2016). Funding value adjustments. ssrn.2746010.

Armakola, A. and J.-P. Laurent (2015). CCP resilience and clearing membership. ssrn.2625579.

Armenti, Y., S. Crépey, S. Drapeau, and A. Papapantoleon (2015). Multivariate shortfall risk allocation and systemic risk. arXiv:1507.05351.

Arnsdorf, M. (2012). Quantification of central counterparty risk. Journal of Risk Management in Financial Institutions 5(3), 273-287.

Avellaneda, M. and R. Cont (2013). Close-out risk evaluation (CORE): A new risk management approach for central counterparties. ssrn.2247493.

Bank for International Settlements (2005). The application of Basel II to trading activities and the treatment of double default effects. http://www.bis.org/publ/bcbs116.pdf.

Basel Committee on Banking Supervision (2005). An explanatory note on the Basel II IRB risk weight functions. http://www.bis.org/bcbs/irbriskweight.pdf.

Basel Committee on Banking Supervision (2011). Basel III: A global regulatory framework for more resilient banks and banking systems. http://www.bis.org/publ/bcbs189.pdf.

Basel Committee on Banking Supervision (2014). Capital requirements for bank exposures to central counterparties. http://www.bis.org/publ/bcbs282.pdf.

Bichuch, M., A. Capponi, and S. Sturm (2016). Arbitrage-free XVA. Mathematical Finance. Forthcoming (preprint version available as ssrn2820257).

Bielecki, T. R. and M. Rutkowski (2015). Valuation and hedging of contracts with funding costs and collateralization. SIAM Journal on Financial Mathematics 6, 594-655.

Brigo, D., M. Morini, and A. Pallavicini (2013). Counterparty Credit Risk, Collateral and Funding: With Pricing Cases For All Asset Classes. Wiley.

Brigo, D. and A. Pallavicini (2014). Nonlinear consistent valuation of CCP cleared or CSA bilateral trades with initial margins under credit, funding and wrong-way risks. Journal of Financial Engineering 1, 1-60.

Burgard, C. and M. Kjaer (2012). The FVA debate: In theory and practice. http://ssrn.com/abstract $=2157634$.

Capponi, A. and W. Cheng (2016). Optimal clearinghouse collateral requirements. ssrn.2669304.

Cont, R. and T. Kokholm (2014). Central clearing of OTC derivatives: bilateral vs multilateral netting. Statistics and Risk Modeling 31(1), 3-22.

Cont, R., R. Mondescu, and Y. Yu (2011). Central clearing of interest rate swaps: a comparison of offerings. ssrn.1783798. 
Cont, R., E. Santos, and A. Moussa (2013). Network structure and systemic risk in banking systems. In J.-P. Fouque and J. Langsam (Eds.), Handbook of Systemic Risk, pp. 327-368. Cambridge University Press.

Crépey, S., T. R. Bielecki, and D. Brigo (2014). Counterparty Risk and Funding: A Tale of Two Puzzles. Chapman \& Hall/CRC Financial Mathematics Series.

Crépey, S. and T. M. Nguyen (2016). Nonlinear Monte Carlo schemes for counterparty risk on credit derivatives. In Challenges in Derivatives Markets, Springer Proceedings in Mathematics, pp. 53-82. Springer.

Crépey, S. and S. Song (2015). BSDEs of counterparty risk. Stochastic Processes and their Applications 125(8), 3023-3052.

Crépey, S. and S. Song (2016). Counterparty risk and funding: Immersion and beyond. Finance and Stochastics 20(4), 901-930. Long preprint version available as hal-00989062, version 2.

Cruz-Lopez, J., J. H. Harris, C. Hurlin, and C. Perignon (2015). CoMargin. Journal of Financial and Quantitative Analysis. Forthcoming.

Duffie, D. (2010). How big banks fail and what to do about it. Princeton University Press.

Duffie, D. (2014). Resolution of failing central counterparties. Working paper no. 3256, Graduate School of Business, Stanford University.

Duffie, D., M. Scheicher, and G. Vuillemey (2015). Central clearing and collateral demand. Journal of Financial Economics 116(2), 237-256.

Duffie, D. and H. Zhu (2011). Does a central clearing counterparty reduce counterparty risk? Review of Asset Pricing Studies 1, 74-95.

Eisenberg, L. and T. H. Noe (2001). Systemic risk in financial systems. Management Science 47(2), 236-249.

European Parliament (2012). Regulation (EU) no 648/2012 of the European parliament and of the council of 4 July 2012 on OTC derivatives, central counterparties and trade repositories. Official Journal of the European Union. http://eur-lex.europa.eu/legalcontent/EN/TXT/PDF/?uri=CELEX:32012R0648\&from=EN.

Fenn, G. and P. Kupiec (1993). Prudential margin policy in a futures-style settlement system. Journal of Futures Markets 13(4), 389-408.

Fujii, M. and A. Takahashi (2012a). Analytical approximation for non-linear FBSDEs with perturbation scheme. International Journal of Theoretical and Applied Finance 15(5), 1250034 (24 pages).

Fujii, M. and A. Takahashi (2012b). Perturbative expansion of FBSDE in an incomplete market with stochastic volatility. Quarterly Journal of Finance 2(3), 1250015 (24 pages).

Ghamami, S. (2015). Static models of central counterparty risk. International Journal of Financial Engineering 02, 1550011-1 to 36. Preprint version available as ssrn.2390946.

Ghamami, S. and P. Glasserman (2016). Does OTC derivatives reform incentivize central clearing? ssrn.2819714.

Gobet, E. and S. Pagliarani (2015). Analytical approximations of BSDEs with nonsmooth driver. SIAM Journal on Financial Mathematics 6, 919-958. 
Green, A., C. Kenyon, and C. Dennis (2014). KVA: capital valuation adjustment by replication. Risk Magazine, December. Preprint version available under the title "KVA: capital valuation adjustment" as ssrn.620454.

He, S.-W., J.-G. Wang, and J.-A. Yan (1992). Semimartingale Theory and Stochastic Calculus. CRC Press.

Henry-Labordère, P. (2012). Cutting CVA's complexity. Risk Magazine, July 67-73.

Hull, J. and A. White (2012). The FVA debate, followed by The FVA debate continued. Risk Magazine, July and October.

Knott, R. and A. Mills (2002). Modelling risk in central counterparty clearing houses: a review. Bank of England Financial Stability Review (December), 162-174.

Kruse, T. and A. Popier (2016). BSDEs with monotone generator driven by Brownian and Poisson noises in a general filtration. Stochastics: An International Journal of Probability and Stochastic Processes 88(4), 491-539.

Kupiec, P. and P. White (1996). Regulatory competition and the efficiency of alternative derivative product margining systems. Journal of Futures Markets 16(8), 943-968.

Levels, A. and J. Capel (2012). Is collateral becoming scarce? Evidence for the euro area. Journal of Financial Market Infrastructures 1(1), 29-53.

Menkveld, A. J. (2016). Crowded trades: an overlooked systemic risk for central clearing counterparties. ssrn.2422250.

Pallavicini, A. and D. Brigo (2013). Interest-rate modelling in collateralized markets: multiple curves, credit-liquidity effects, CCPs. arXiv:1304.1397.

Singh, M. (2010). Collateral, netting and systemic risk in the OTC derivatives market. Technical report, IMF working paper WP/10/99.

Singh, M. and J. Aitken (2009). Counterparty risk, impact on collateral flows, and role for central counterparties. Technical report, IMF working paper WP/09/173. 